\title{
Interval-Valued and Intuitionistic Fuzzy Mathematical Morphologies as Special Cases of $\mathbb{L}$-Fuzzy Mathematical Morphology
}

\author{
Peter Sussner ${ }^{\star}$ • Mike Nachtegael • Tom Mélange • Glad Deschrijver • \\ Estevão Esmi · Etienne Kerre
}

the date of receipt and acceptance should be inserted later

\begin{abstract}
Mathematical morphology (MM) offers a wide range of tools for image processing and computer vision. MM was originally conceived for the processing of binary images and later extended to gray-scale morphology. Extensions of classical binary morphology to gray-scale morphology include approaches based on fuzzy set theory that give rise to fuzzy mathematical morphology (FMM). From a mathematical point of view, FMM relies on the fact that the class of all fuzzy sets over a certain universe forms a complete lattice. Recall that complete lattices provide for the most general framework in which MM can be conducted.

The concept of $\mathbb{L}$-fuzzy set generalizes not only the concept of fuzzy set but also the concepts of interval-valued fuzzy set and Atanassov's intuitionistic fuzzy set. In addition, the class of $\mathbb{L}$-fuzzy sets forms a complete lattice whenever the underlying set $\mathbb{L}$ constitutes a complete lattice. Based on these observations, we develop a general approach towards $\mathbb{L}$-fuzzy mathematical morphology in this paper. Our focus is in particular on the construction of connectives for interval-valued and intuitionistic fuzzy mathematical morphologies that arise as special, isomorphic cases of $\mathbb{L}$-fuzzy MM. As an application of these ideas, we generate a combination of some well-known medical image reconstruction
\end{abstract}

Grants or other notes.

Peter Sussner and Estevão Esmi

University of Campinas, Department of Applied Mathematics, Campinas, SP, 13083-859, Brazil

Tel.: +55-19-3521 5959

Fax: +55-19-3289-5766

E-mail: sussner,ra050652@ime.unicamp.br

Mike Nachtegael, Tom Mélange, Glad Deschrijver, and Etienne Kerre Ghent University, Department of Applied Mathematics and Computer Science, Krijgslaan 281 - S9, 9000 Ghent, Belgium

Tel.: +32-9-264 4765

Fax: +32-9-264 4995

E-mail: Mike.Nachtegael,Tom.Melange,Glad.Deschrijver,Etienne.Kerre @UGent.be techniques in terms of interval-valued fuzzy image processing.

Keywords Mathematical morphology · complete lattice · $\mathbb{L}$-fuzzy sets · interval-valued fuzzy sets · Atanassov's intuitionistic fuzzy sets $\cdot \mathbb{L}$-fuzzy mathematical morphology $\cdot \mathbb{L}$-fuzzy connectives $\cdot$ inclusion measure $\cdot$ duality negation $\cdot$ adjunction

\section{Introduction}

Recently, Type-2 and intuitionistic fuzzy set theories have become increasingly important in applications in rule-based systems and approximate reasoning [26,83,84]. Both Type2 and intuitionistic fuzzy set theory extend Zadeh's fuzzy set theory $[1,88,89]$.

Recall that a conventional or Type-1 fuzzy set has crisp membership degrees that reside in the unit interval $[0,1]$. In contrast, a Type-2 fuzzy set allows for membership degrees that are Type- 1 fuzzy sets on the universe $[0,1]$, i.e., a Type- 2 fuzzy set $\tilde{A}$ represents a function from a set $X$ to the class of Type- 1 fuzzy sets on $[0,1]$. An element $x \in X$ is mapped to a Type-1 fuzzy set $\tilde{A}(x)$ which is called the secondary membership function at $x$. A particular class of Type-2 fuzzy sets arises if, for every $x \in X$, the secondary membership function $\tilde{A}(x)$ equals the characteristic function of an interval $I_{x} \subseteq[0,1]$. In this case we speak of interval Type-2 fuzzy sets [51]. For computational reasons, most practical applications of Type-2 fuzzy sets are indeed only concerned with the subclass of interval Type-2 fuzzy sets $[52,53,84]$. For most authors interval Type-2 fuzzy sets and intervalvalued fuzzy sets (IVFSs) [34,42,66,89] are two equivalent concepts although there are some conceptual differences regarding notions such as cardinality and support of interval Type-2 fuzzy sets and interval-valued fuzzy sets [60]. Recall that an interval-valued fuzzy set corresponds to a map- 
ping $A$ from $X$ into the class of intervals $\left[\mu_{1}, \mu_{2}\right] \subseteq[0,1]$. Thus, $A(x)=\left[\mu_{1}(x), \mu_{2}(x)\right]$ for every $x \in X$. Evidently, if $\mu_{1}(x)$ equals $\mu_{2}(x)$ for all $x \in X$ then the interval fuzzy set reduces to a (Type-1) fuzzy set. Interval-valued fuzzy sets have been used successfully by J. M. Mendel to implement Zadeh's paradigm of computing with words [50].

Intuitionistic fuzzy sets (IFSs) generalize Zadeh's original definition by defying the law of the excluded middle which claims that if $x$ belongs to a degree $\mu$ to a fuzzy set then $x$ does not belong to this fuzzy set to the extent $\nu=1-\mu[1,3]$. In IFS theory, the degree of membership of $x$ and the degree of non-membership of $x$ do not have to add up to 1 . Instead, IFS theory only requires that the pair consisting of the membership degree $\mu$ and the non-membership degree $\nu$ of $x$ in a satisfies the inequality $\mu+\nu \leq 1$.

Atanassov coined the technical term "intuitionistic fuzzy set" since intuitionistic logic also rejects the law of the excluded middle [2]. As Dubois et al. have pointed out [31], this terminology is unfortunate and misleading. Therefore, these prominent researchers have advocated a change in terminology from "intuitionistic fuzzy set" to "bipolar fuzzy set" in view of the fact that the term "bipolarity" captures the separate handling of positive and negative aspects of information. However, we would like to stress that the concept of "bipolar fuzzy set" had already been previously introduced by W.-R. Zhang et al. [91,92]. Although Zhang's work on bipolar fuzzy sets is also concerned with two sides of a matter or positive and negative aspects of information, his bipolar fuzzy sets are formally different from Atanassov's IFSs. For instance, the bipolar fuzzy space is given by $[-1,0] \times$ $[0,1]$ which represents the set of pairs having a negative side as well as a positive side. Based on these observations, we prefer to adhere to the nomenclature "intuitionistic fuzzy set" so as to be in agreement with the terminology that prevails in the literature and in the premier conferences on fuzzy sets and systems.

This paper investigates a number of theoretical aspects of $\mathbb{L}$-fuzzy mathematical morphology. Unlike previous papers on interval-valued and intuitionistic fuzzy mathematical morphology $[10,11,58,59]$, this paper treats intervalvalued and intuitionistic FMMs as special cases of $\mathbb{L}$-fuzzy MM. This approach not only allows for a top-down view of the corresponding mathematical frameworks but also for the construction of $\mathbb{L}$-fuzzy MMs for other particular instances of complete lattices $\mathbb{L}$.

Special attention is given to extensions of FMM that are known as interval-valued and intuitionistic FMMs in which case the complete lattices in question are denoted by $\mathbb{L}^{I}$ and $\mathbb{L}^{*}[27,59]$. In analogy to the fuzzy case $[56,80]$, we stipulate that a certain approach towards interval-valued or intuitionistic FMM depends on the choice of interval-valued or intuitionistic fuzzy inclusion and intersection measures that are determined by pairs consisting of an implication and a conjunction on $\mathbb{L}^{I}$ or $\mathbb{L}^{*}$. Each pair of connectives should be linked in terms of a duality relationship of adjunction and/or negation. In view of these considerations, Section 4 is devoted to the construction of interval-valued or intuitionistic fuzzy connectives that may serve as a basis for a particular approach towards interval-valued or intuitionistic FMM. In Section 5, we consider a specific pair of intervalvalued fuzzy connectives consisting of an $t$-norm and an implication on $\mathbb{L}^{I}$ that are both adjoint and dual with respect to the standard negation on $\mathbb{L}^{I}$. This $t$-norm and this implication on $\mathbb{L}^{I}$ give rise to respectively an interval-valued fuzzy dilation and an interval-valued fuzzy erosion that can be employed to compute the interval-valued morphological gradient of interval-valued fuzzy images. Indeed, we adopt this strategy for generating the interval-valued morphological gradient of an interval-valued fuzzy representation of a combination of results produced by three well-known medical image reconstruction methods. After some appropriate post-processing, the mean of the upper and lower envelopes of the interval-valued morphological gradient image is transformed using the watershed segmentation algorithm. The reconstructed images obtained from the individual reconstruction algorithms are segmented in a similar way. The segmentation results produced by the individual methods and by the interval-valued combination of these methods can be visually compared by taking the segmention of the original image as the ground truth.

The paper is organized as follows. The first section deals with general mathematical concepts. Specifically, after reviewing the mathematical background of MM on complete lattices, we investigate the properties of $\mathbb{L}$-fuzzy logical connectives that we employ to derive $\mathbb{L}$-fuzzy inclusion and intersection measures. In Section 3, we introduce the general mathematical framework of $\mathbb{L}$-fuzzy MM as a generalization of FMM. Section 4 focusses on the special cases of intervalvalued and intuitionistic FMMs, in particular on the construction of the underlying interval-valued and intuitionistic fuzzy logical connectives. Section 5 applies the concepts of interval-valued FMM that we developped in the previous sections to generate a combination of distinct medical image reconstruction algorithms and to process the interval-valued images that correspond to this combination. Finally, we finish the paper with some concluding remarks and suggestions for further research.

\section{General Mathematical Concepts}

\subsection{The Complete Lattice Framework of Mathematical Morphology}

Mathematical Morphology (MM) is a theory that uses concepts from set theory, geometry and topology to analyze geometrical structures in an image [37,48,68,69]. MM has 
found wide-spread applications over the entire imaging spectrum $[16,35,36,44,62,73,74]$. MM was originally invented in the early 1960s by Georges Matheron and Jean Serra as a tool for the automatic analysis of binary images [47,67]. After Sternberg and Serra extended MM to gray-scale images $[68,75]$, Serra observed that complete lattice theory represents the appropriate algebraic framework for MM [37,64, 69]. Recent research results of Heijmans and Keshet have extended this framework to complete inf-semilattices [38]. Recent expositions on the lattice-theoretical framework of MM include [14,65].

The fact that the unit interval $[0,1]$ represents a complete lattice has played a crucial role in the development of fuzzy mathematical morphology (FMM) which can be viewed as an extension of binary MM to gray-scale MM [20-24,32, $41,46,56,59,80]$. In this context, note that a fuzzy set corresponds to an $\mathbb{L}$-fuzzy set where $\mathbb{L}=[0,1]$ [33]. The complete lattice setting allows for an algebraic definition of the elementary operators of MM, namely erosion, dilation, antierosion, and anti-dilation [37,69]. An (algebraic) erosion is defined as an operator $\varepsilon$ from a complete lattice $\mathbb{L}$ to a complete lattice $\mathbb{M}$ that commutes with the infimum operation. Similarly, an (algebraic) dilaton is defined as an operator $\mathbb{L} \rightarrow \mathbb{M}$ that commutes with the supremum operation. Formally, an erosion is an operator $\varepsilon: \mathbb{L} \rightarrow \mathbb{M}$ satisfying the left side of Equation 1 and a dilation $\delta: \mathbb{L} \rightarrow \mathbb{M}$ is an operator $\varepsilon: \mathbb{L} \rightarrow \mathbb{M}$ satisfying the right side of Equation 1 .

$$
\varepsilon(\bigwedge Y)=\bigwedge_{y \in Y} \varepsilon(y), \delta(\bigvee Y)=\bigvee_{y \in Y} \delta(y) \forall Y \subseteq \mathbb{L}
$$

Anti-erosions and anti-dilations arise from negations, erosions, and dilations [85]. Recall that a negation on a lattice is an involutive bijection that reverses the partial ordering.

Two important notions of duality permeate MM: adjunction and negation. The operators of erosion and dilation can be linked by means of negation. Let $\Psi$ be an operator mapping a complete lattice $\mathbb{L}$ into a complete lattice $\mathbb{M}$ and let $\nu_{\mathbb{L}}$ and $\nu_{\mathbb{M}}$ be negations on $\mathbb{L}$ and $\mathbb{M}$, respectively. The operator $\Psi^{\nu}$ given by

$\Psi^{\nu}(x)=\nu_{\mathbb{M}}\left(\Psi\left(\nu_{\mathbb{L}}(x)\right)\right) \quad \forall x \in \mathbb{L}$,

is called the negation of $\Psi$ (with respect to $\nu_{\mathbb{L}}$ and $\nu_{\mathbb{M}}$ ). Thus, we have that the negation of an erosion is a dilation, and vice-versa [37].

Several prominent researchers [24,39,37,46,64] consider adjunction to be the most important notion of duality in MM. Consider two arbitrary operators $\delta: \mathbb{L} \rightarrow \mathbb{M}$ and $\varepsilon: \mathbb{M} \rightarrow \mathbb{L}$ for some complete lattices $\mathbb{L}$ and $\mathbb{M}$. We say that the pair $(\varepsilon, \delta)$ forms an adjunction or that $\varepsilon$ and $\delta$ are adjoint if and only if we have

$\delta(x) \leq y \Leftrightarrow x \leq \varepsilon(y) \quad \forall x \in \mathbb{L}, \forall y \in \mathbb{M}$.
The following well-known properties of adjunctions [37, 69] will turn out to be extremely useful throughout the paper. Specifically, the following statements are valid for mappings $\delta: \mathbb{L} \rightarrow \mathbb{M}$ and $\varepsilon: \mathbb{M} \rightarrow \mathbb{L}$, where $\mathbb{L}$ and $\mathbb{M}$ are complete lattices:

1. If $(\varepsilon, \delta)$ is an adjunction then $\varepsilon$ is an erosion and $\delta$ is a dilation.

2. For any dilation $\delta$ there is a unique erosion $\varepsilon$ such that $(\varepsilon, \delta)$ is an adjunction. The adjoint erosion is given by

$$
\varepsilon(y)=\bigvee\{x \in \mathbb{L}: \delta(x) \leq y\},
$$

for every $y \in \mathbb{M}$.

3. For any erosion $\varepsilon$ there is a unique dilation $\delta$ such that $(\varepsilon, \delta)$ is an adjunction. The adjoint dilation is given by

$$
\delta(x)=\bigwedge\{y \in \mathbb{M}: \varepsilon(y) \geq x\}
$$

for every $x \in \mathbb{L}$.

The preceding observations clarify that there is a unique erosion that can be associated with a certain dilation, and vice-versa, in terms of either negation or adjunction. Adjoint pairs of erosions and dilations also give rise to openings and closings [37].

\subsection{Logical Operators on a Complete Lattice and Relationships of Duality}

Fuzzy logical operators are well-known extensions of Boolean logical operators. These operators associate elements of $[0,1]^{2}$ with elements of $[0,1]$ or - in the case of negation map the unit interval $[0,1]$ into $[0,1]$. Instead of the complete lattice $[0,1]$, we can take any complete lattice $\mathbb{L}$ and define logical operators on $\mathbb{L}$ as $\mathbb{L}^{2} \rightarrow \mathbb{L}$ or $\mathbb{L} \rightarrow \mathbb{L}$ mappings. This strategy yields the following definitions [25]:

Definition 1 Let $\mathbb{L}$ be a complete lattice with smallest element $0_{\mathbb{L}}$ and largest element $1_{\mathbb{L}}$.

- A conjunction on $\mathbb{L}$ or $\mathbb{L}-$ fuzzy conjunction is defined as an increasing mapping $C: \mathbb{L} \times \mathbb{L} \rightarrow \mathbb{L}$ that satisfies $C\left(0_{\mathbb{L}}, 0_{\mathbb{L}}\right)=C\left(0_{\mathbb{L}}, 1_{\mathbb{L}}\right)=C\left(1_{\mathbb{L}}, 0_{\mathbb{L}}\right)=0_{\mathbb{L}}$ and $C\left(1_{\mathbb{L}}, 1_{\mathbb{L}}\right)=1_{\mathbb{L}}$. In particular, a commutative and associative $\mathbb{L}$-fuzzy conjunction $T: \mathbb{L} \times \mathbb{L} \rightarrow \mathbb{L}$ that satisfies $T\left(x, 1_{\mathbb{L}}\right)=x$ for every $x \in \mathbb{L}$ is called triangular norm or simply $t$-norm on $\mathbb{L}$.

- A disjunction on $\mathbb{L}$ or $\mathbb{L}$-fuzzy disjunction is an increasing mapping $D: \mathbb{L} \times \mathbb{L} \rightarrow \mathbb{L}$ that satisfies $D\left(0_{\mathbb{L}}, 0_{\mathbb{L}}\right)=$ $0_{\mathbb{L}}$ and $D\left(0_{\mathbb{L}}, 1_{\mathbb{L}}\right)=D\left(1_{\mathbb{L}}, 0_{\mathbb{L}}\right)=D\left(1_{\mathbb{L}}, 1_{\mathbb{L}}\right)=1_{\mathbb{L}}$. In particular, a commutative and associative $\mathbb{L}$-fuzzy disjunction $S: \mathbb{L} \times \mathbb{L} \rightarrow \mathbb{L}$ that satisfies $S\left(x, 0_{\mathbb{L}}\right)=x$ for every $x \in\left[0_{\mathbb{L}}, 1_{\mathbb{L}}\right]$ is called triangular co-norm or s-norm on $\mathbb{L}$. 
- An operator $I: \mathbb{L} \times \mathbb{L} \rightarrow \mathbb{L}$ that is decreasing in the first argument and that is increasing in the second argument is called an implication on $\mathbb{L}$ or $\mathbb{L}$-fuzzy implication if the equations $I\left(0_{\mathbb{L}}, 0_{\mathbb{L}}\right)=I\left(0_{\mathbb{L}}, 1_{\mathbb{L}}\right)=I\left(1_{\mathbb{L}}, 1_{\mathbb{L}}\right)=1_{\mathbb{L}}$ and $I\left(1_{\mathbb{L}}, 0_{\mathbb{L}}\right)=0_{\mathbb{L}}$ are satisfied.

Recall that we already reviewed the concept of negation on a complete lattice $\mathbb{L}$ in the previous section. We will also speak of an $\mathbb{L}$-fuzzy negation. The $\mathbb{L}$-fuzzy connectives on the complete lattice $[0,1]$ are known as fuzzy conjunctions, disjunctions, implications, $t$-norms, $s$-norms, and negations.

A logical connective can be associated with another logical connective on $\mathbb{L}$ in terms of a duality relationship of negation or adjunction. Let us introduce the following definitions that extend the respective definitions for the complete lattice $[0,1]$.

Definition 2 Let $C$ be a conjunction, $D$ a disjunction, and $I$ an implication on $\mathbb{L}$. Moreover, let $N$ be a negation on $\mathbb{L}$.

- We say that $C$ and $D$ are dual (operators) with respect to $N$ if and only if the following equation holds for every $x, y \in \mathbb{L}$ :

$$
C(x, y)=N(D(N(x), N(y)) .
$$

- We say that $C$ and $I$ are dual (operators) with respect to $N$ if and only if $C(z, \cdot)$ and $I(z, \cdot)$ are dual with respect to $N$ for all $z \in \mathbb{L}$. In this case, we have the following equation for all $x, z \in \mathbb{L}$ :

$$
C(z, x)=N(I(z, N(x))) .
$$

- We say that $I$ and $D$ are dual (operators) with respect to $N$ if and only if the following equation holds for every $x, y \in \mathbb{L}$ :

$$
I(x, y)=D(N(x), y)
$$

In contrast to a pair of operators that are dual with respect to negation, a pair of adjoint operators $\varepsilon$ and $\delta$ has the advantage that $\varepsilon$ is guaranteed to represent an erosion and that $\delta$ is guaranteed to represent a dilation in the latticealgebraic sense of Equation 1.

Definition 3 Let $\mathbb{L}$ be a complete lattice. An $\mathbb{L}$-fuzzy implication $I$ and an $\mathbb{L}$-fuzzy conjunction $C$ form an adjunction if and only if $I(z, \cdot)$ and $C(z, \cdot)$ form an adjunction for every $z \in \mathbb{L}$. In this case, the following statement is true for all $x, y, z \in \mathbb{L}$ :

$C(z, x) \leq y \Leftrightarrow x \leq I(z, y)$.

Note that if an implication $I$ and a conjunction $C$ on $\mathbb{L}$ are adjoint then $C(z, \cdot)$ is a dilation and that $I(z, \cdot)$ is an erosion for every $z \in \mathbb{L}$. Also note that we can employ Equation 4 to form the adjoint erosion $I(z,$.$) of a dilation$ $C(z,$.$) where C$ is a conjunction on $\mathbb{L}$. Conversely, we can employ Equation 5 to form the adjoint dilation $C(z,$.$) of$ an erosion $I(z,$.$) where I$ is a implication on $\mathbb{L}$. The conditions stated in Theorem 1 and 2 guarantee that the respective adjoint operators are $\mathbb{L}$-fuzzy implications and conjunctions. The definition of $I_{C}$ in Theorem 1 generalizes the definitions of intuitionistic and interval-valued $R$-implicators provided in [18].

Theorem 1 Let $C$ be an $\mathbb{L}$-fuzzy conjunction. Suppose that $I_{C}: \mathbb{L}^{2} \rightarrow \mathbb{L}$ is defined as follows:

$I_{C}(z, y)=\bigvee\{x \in \mathbb{L}: C(z, x) \leq y\} \forall z, y \in \mathbb{L}$

The following statements hold true.

1. The mapping $I_{C}$ is decreasing in the first argument, increasing in the second argument, and satisfies the conditions

$$
I_{C}\left(0_{\mathbb{L}}, 0_{\mathbb{L}}\right)=I_{C}\left(0_{\mathbb{L}}, 1_{\mathbb{L}}\right)=I_{C}\left(1_{\mathbb{L}}, 1_{\mathbb{L}}\right)=1_{\mathbb{L}} .
$$

2. The mapping $I_{C}$ represents an $\mathbb{L}$-fuzzy implication if and only if $C\left(1_{\mathbb{L}}, x\right)>0_{\mathbb{L}}$ for all $x \in \mathbb{L} \backslash\left\{0_{\mathbb{L}}\right\}$. In this case, the implication $I_{C}$ is referred to as the $R$-implication of C.

Conversely, we are able to derive an $\mathbb{L}$-fuzzy conjunction $C_{I}$ from an $\mathbb{L}$-fuzzy implication $I$ under certain conditions that are stated in the following theorem.

Theorem 2 Let I be an $\mathbb{L}$-fuzzy implication. Suppose that $C_{I}:(\mathbb{L})^{2} \rightarrow \mathbb{L}$ is defined as follows:

$C_{I}(z, x)=\bigwedge\{y \in \mathbb{L}: I(z, y) \geq x\} \forall z, x \in \mathbb{L}$.

The following statements hold true.

1. The mapping $C_{I}$ is increasing and satisfies the conditions

$$
C_{I}\left(0_{\mathbb{L}}, 0_{\mathbb{L}}\right)=C_{I}\left(0_{\mathbb{L}}, 1_{\mathbb{L}}\right)=C_{I}\left(1_{\mathbb{L}}, 0_{\mathbb{L}}\right)=0_{\mathbb{L}} .
$$

2. The mapping $C_{I}$ represents an $\mathbb{L}$-fuzzy conjunction if and only if $I\left(1_{\mathbb{L}}, y\right)<1_{\mathbb{L}}$ for all $y \in \mathbb{L} \backslash\left\{1_{\mathbb{L}}\right\}$. In this case, we say that $C_{I}$ is the $R$-conjunction of $I$.

The following theorem deals with successive applications of Equations 10 and 12

Theorem 3 Let $C$ be an $\mathbb{L}$-fuzzy conjunction that satisfies $C\left(1_{\mathbb{L}}, x\right)>0_{\mathbb{L}}$ for all $x \neq 0_{\mathbb{L}}$. If $I_{C}$, the $R$-implication of $C$, satisfies $I_{C}\left(1_{\mathbb{L}}, y\right)<1_{\mathbb{L}}$ for all $y \neq 1_{\mathbb{L}}$ then the $R$ conjunction of $I_{C}$ is bounded from above by $C$.

Similarly, let $I$ be an $\mathbb{L}$-fuzzy implication that satisfies $I\left(1_{\mathbb{L}}, y\right)<1_{\mathbb{L}}$ for all $y \neq 1_{\mathbb{L}}$. If $C_{I}$, the $R$-conjunction of $I$, satisfies $C_{I}\left(1_{\mathbb{L}}, x\right)>0_{\mathbb{L}}$ for all $x \neq 0_{\mathbb{L}}$ then the $R$ implication of $C_{I}$ is bounded from below by $I$. 
Note that the inequalities $I\left(1_{\mathbb{L}}, y\right)<1_{\mathbb{L}}$ for all $y \neq 1_{\mathbb{L}}$ are in particular satisfied for the class of $\mathbb{L}$-fuzzy implications such that $I\left(1_{\mathbb{L}}, y\right)=y$ for all $y \in \mathbb{L}$. Similarly, the inequalities $C\left(1_{\mathbb{L}}, x\right)>0_{\mathbb{L}}$ for all $x \neq 0_{\mathbb{L}}$ are in particular satisfied for the class of $\mathbb{L}$-fuzzy conjunctions such that $C\left(1_{\mathbb{L}}, x\right)=x$ for all $x \in \mathbb{L}$. The latter class encompasses the class of $\mathbb{L}$-fuzzy $t$-norms. Extending well-known nomenclature, we say that an application of Equation 10 to an $\mathbb{L}$-fuzzy $t$-norm $T$ results in the $R$-implication of $T$.

Theorems 1 and 2 reveal that some care has to be taken when trying to generate an $\mathbb{L}$-fuzzy implication from an $\mathbb{L}$ fuzzy conjunction by means of Equation 10 or an $\mathbb{L}$-fuzzy conjunction from an $\mathbb{L}$-fuzzy implication by means of Equation 12 , respectively. Fortunately, the construction of $\mathbb{L}$-fuzzy operators from another $\mathbb{L}$-fuzzy operator using negation entails no complications:

Theorem 4 Let $N$ be a negation on $\mathbb{L}$. Suppose that the $\mathbb{L}-$ fuzzy operators $C$ and I are related in terms of Equation 7 , i.e., $C$ and $I$ are dual with respect to $N$. We have that $C$ is a conjunction on $\mathbb{L}$ if and only if $I$ is an implication on $\mathbb{L}$. Similar statements hold true for Equations 6 and 8.

Consider Equation 8 in the special case where the disjunction is an $s$-norm on $\mathbb{L}$ that is denoted using the symbol $S$. Extending the notions of strong implications or, for short, $S$-implications both in conventional as well as in intervalvalued and intuitionistic fuzzy set theories [18], the implicator $I$ given by $I(x, y)=S(N(x), y)$ for all $x, y \in \mathbb{L}$ is called an $S$-implication on $\mathbb{L}$.

\subsection{L-Fuzzy Inclusion and Intersection Measures}

In Section 2.1, we presented the formal definitions of an erosion and a dilation within the complete lattice framework of MM. As mentioned before, these and other connections to lattice algebra were discovered by Serra and Heijmans only at later stages of the development of MM [37,69]. The origins of MM lie in certain types of applications of set theory and of geometry to image processing. In binary MM, an image $\mathbf{a}: \mathbf{X} \rightarrow\{0,1\}$ is viewed as a subset of $\mathbf{X}$ which can be assumed to be either the Euclidean space $\mathbb{R}^{d}$ or the digital space $\mathbb{Z}^{d}$. The fundamental operation of binary erosion yields the set of points for which a translated structuring element is contained in the input image [68]. The threshold approach and the umbra approach to grayscale MM employ straightforward extensions of this basic idea to the grayscale case $[68,75]$. In a similar vein, the fundamental operation of dilation is defined in terms of intersection of sets.

Thus, the concepts of set inclusion and set intersection lie at the root of MM. Researchers in fuzzy mathematical morphology (FMM) have devised fuzzy inclusion and intersection measures by relaxing the notions of crisp inclusion and intersection measure $[7,45,71,72,88]$. Large classes of fuzzy inclusion measures and fuzzy intersection measures can be constructed in terms of fuzzy implications and conjunctions [80].

The notion of $\mathbb{L}$-fuzzy set on a universe $U$ was introduced by Goguen as natural extension of the notion of fuzzy set. An $\mathbb{L}$-fuzzy set on the universe $\mathbf{X}$ is an $\mathbf{X} \rightarrow \mathbb{L}$ mapping where $\mathbb{L}$ is a complete lattice that is equipped with a negation on $\mathbb{L}$ [33]. The class of $\mathbb{L}$-fuzzy sets on the universe $\mathbf{X}$ which is generally denoted using the symbol $\mathcal{F}_{\mathbb{L}}(\mathbf{X})$ encompasses the classes of interval-valued fuzzy sets on $\mathbf{X}$ and intuitionistic fuzzy sets on $\mathbf{X}$. In Sections 4 and 5, we will discuss these special cases. Note that $\mathcal{F}_{\mathbb{L}}(\mathbf{X})$ represents a complete lattice whose partial ordering is induced by the partial ordering on the complete lattice $\mathbb{L}$, i.e. for $\mathbf{a}, \mathbf{b} \in \mathcal{F}_{\mathbb{L}}(\mathbf{X})$ we have $\mathbf{a} \leq \mathbf{b}$ if and only if $\mathbf{a}(\mathbf{x}) \leq \mathbf{b}(\mathbf{x}) \forall \mathbf{x} \in \mathbf{X}$.

Generalizing the construction of approaches towards FMM $[56,80]$, this paper presents a general scheme for constructing approaches towards $\mathbb{L}$-fuzzy MM. To this end, we need to introduce a notion of $\mathbb{L}$-fuzzy inclusion measure that can be employed to define the concept of $\mathbb{L}$-fuzzy erosion. Clearly, an $\mathbb{L}$-fuzzy inclusion measure $I n c_{\mathbb{L}}$ should act on $\mathcal{F}_{\mathbb{L}}(\mathbf{X}) \times \mathcal{F}_{\mathbb{L}}(\mathbf{X})$ and should extend the notions of intuitionistic, interval-valued, and conventional fuzzy inclusion measures. As Cornelis and Kerre have explained, an intuitionistic fuzzy inclusion measure should associate a pair of IFSs with an element of $\mathbb{L}^{*}=\left\{(\mu, \nu) \in[0,1]^{2}: \mu+\nu \leq 1\right\}$ [19] and therefore an interval-valued fuzzy inclusion measure should associate a pair of IVFSs with an element of $\mathbb{L}^{I}=\{[x, y] \subseteq[0,1]\}$. These considerations lead us to the following definition of $\mathbb{L}$-fuzzy inclusion measure:

Definition 4 An $\mathbb{L}$-fuzzy inclusion measure defined as a function $\operatorname{Inc}_{\mathbb{L}}: \mathcal{F}_{\mathbb{L}}(\mathbf{X}) \times \mathcal{F}_{\mathbb{L}}(\mathbf{X}) \rightarrow \mathbb{L}$ that satisfies the following properties for all $\mathbf{a}, \mathbf{b} \in \mathcal{P}_{\mathbb{L}}(\mathbf{X})$, where $\mathcal{P}_{\mathbb{L}}(\mathbf{X})=\{\mathbf{a} \in$ $\mathcal{F}_{\mathbb{L}}(\mathbf{X}): \mathbf{a}(\mathbf{x})=0_{\mathbb{L}}$ or $\left.\mathbf{a}(\mathbf{x})=1_{\mathbb{L}} \forall \mathbf{x} \in \mathbf{X}\right\}$.

$\mathbf{a} \leq \mathbf{b} \Rightarrow \operatorname{Inc_{\mathbb {L}}}(\mathbf{a}, \mathbf{b})=1_{\mathbb{L}}$ and $\mathbf{a} \not \mathbf{b} \Rightarrow I n c_{\mathbb{L}}(\mathbf{a}, \mathbf{b})=0_{\mathbb{L}}$.

Extending the concept of fuzzy intersection measure to the $\mathbb{L}$-fuzzy domain leads to the following definition:

Definition 5 An $\mathbb{L}$-fuzzy intersection measure is a function $S e c_{\mathbb{L}}: \mathcal{F}_{\mathbb{L}}(\mathbf{X}) \times \mathcal{F}_{\mathbb{L}}(\mathbf{X}) \rightarrow \mathbb{L}$ that satisfies the following properties for all $\mathbf{a}, \mathbf{b} \in \mathcal{P}_{\mathbb{L}}(\mathbf{X})$.

$\mathbf{a} \wedge \mathbf{b} \neq 0_{\mathcal{F}_{\mathbb{L}}(\mathbf{X})} \Rightarrow \operatorname{Sec}_{\mathbb{L}}(\mathbf{a}, \mathbf{b})=1_{\mathbb{L}}$,
$\mathbf{a} \wedge \mathbf{b}=0_{\mathcal{F}_{\mathbb{L}}(\mathbf{X})} \Rightarrow \operatorname{Sec}_{\mathbb{L}}(\mathbf{a}, \mathbf{b})=0_{\mathbb{L}}$.

Evidently, an $\mathbb{L}$-fuzzy implication $I$ gives rise to an $\mathbb{L}$ fuzzy inclusion measure $I n c_{\mathbb{L}}$ and an $\mathbb{L}$-fuzzy conjunction 
$C$ gives rise to an $\mathbb{L}$-fuzzy intersection measure $S e c_{\mathbb{L}}$ if we define $I n c_{\mathbb{L}}$ and $S e c_{\mathbb{L}}$ as follows for all $\mathbf{a}, \mathbf{b} \in \mathcal{F}_{\mathbb{L}}(\mathbf{X})$ :

$$
\begin{aligned}
\operatorname{Inc}_{\mathbb{L}}(\mathbf{a}, \mathbf{b}) & =\bigwedge_{\mathbf{x} \in \mathbf{X}} I(\mathbf{a}(\mathbf{x}), \mathbf{b}(\mathbf{x})) . \\
\operatorname{Sec}_{\mathbb{L}}(\mathbf{a}, \mathbf{b}) & =\bigvee_{\mathbf{x} \in \mathbf{X}} C(\mathbf{a}(\mathbf{x}), \mathbf{b}(\mathbf{x})) .
\end{aligned}
$$

We will refer to the operator $I n c_{\mathbb{L}}$ in Equation 17 as $\mathbb{L}_{\text {- }}$ fuzzy Inf-I inclusion measure and we will refer to the operator $S e c_{\mathbb{L}}$ in Equation 18 as $\mathbb{L}$-fuzzy Sup-C intersection measure. The next section reviews the construction of approaches to fuzzy MM based on fuzzy Inf-I inclusion measures and Sup-C intersection measures. Approaches to $\mathbb{L}$ fuzzy MM arise as obvious extensions of this construction.

\section{From Fuzzy Mathematical Morphology to $\mathbb{L}$-Fuzzy Mathematical Morphology}

\subsection{Some Basic Concepts of Fuzzy Mathematical Morphology}

A certain approach to FMM is determined by certain definitions of fuzzy erosion and dilation since a fuzzy antidilation and anti-erosion can be constructed by combining a fuzzy erosion or a fuzzy dilation with a fuzzy negation. To maintain the consistency with the complete lattice framework for mathematical morphology we say that a function $\varepsilon_{\mathcal{F}}: \mathcal{F}(U) \rightarrow \mathcal{F}(V)$ is a fuzzy erosion if and only if $\varepsilon_{\mathcal{F}}$ is an erosion in the sense of Equation 1. Similarly, we say that an operator $\delta_{\mathcal{F}}: \mathcal{F}(U) \rightarrow \mathcal{F}(V)$ is a fuzzy dilation if and only if it satisfies Equation 1.

Let us leave these purely mathematical considerations aside for a moment. Intuitively speaking, the notion of erosion, dilation respectively, is meant to extract some relevant information on the shape and form of objects by means of a structuring element (SE) [68]. Hence, a fuzzy erosion $\varepsilon_{\mathcal{F}}: \mathcal{F}(\mathbf{X}) \rightarrow \mathcal{F}(\mathbf{X})$, a fuzzy dilation $\delta_{\mathcal{F}}: \mathcal{F}(\mathbf{X}) \rightarrow$ $\mathcal{F}(\mathbf{X})$ respectively, is generally given by a rule that combines an input fuzzy set $\mathbf{x} \in \mathcal{F}(\mathbf{X})$ with an arbitrary, but fixed fuzzy structuring element $\mathbf{s} \in \mathcal{F}(\mathbf{X})$ and generates an output fuzzy set $\mathbf{y} \in \mathcal{F}(\mathbf{X})$. Recall that $\mathbf{s}_{\mathbf{x}}$, the translation of $\mathbf{s}$ by $\mathbf{x}$, and $\overline{\mathbf{s}}$, the reflection of $\mathbf{s}$ around the origin, are defined as follows:

$\mathbf{s}_{\mathbf{x}}(\mathbf{y})=\mathbf{s}(\mathbf{y}-\mathbf{x}), \overline{\mathbf{s}}(\mathbf{y})=\mathbf{s}(-\mathbf{y}), \forall \mathbf{y} \in \mathbf{X}$.

The value $\operatorname{Inc}_{\mathcal{F}}(\mathbf{a}, \mathbf{b})$ can be interpreted as the degree of subsethood or inclusion of the fuzzy set a in the fuzzy set b. Various researchers have presented fuzzy inclusion measures $[7,45,71,72,88]$. A certain fuzzy inclusion measure Inc $c_{\mathcal{F}}$ induces an operator $\mathcal{E}_{\mathcal{F}}: \mathcal{F}(\mathbf{X}) \times \mathcal{F}(\mathbf{X}) \rightarrow \mathcal{F}(\mathbf{X})$ via the following definition [56]:

$\mathcal{E}_{\mathcal{F}}(\mathbf{a}, \mathbf{s})(\mathbf{x})=\operatorname{Inc} c_{\mathcal{F}}\left(\mathbf{s}_{\mathbf{x}}, \mathbf{a}\right)$.
We refer to $\mathcal{E}_{\mathcal{F}}$ as a fuzzy erosion if $\operatorname{Inc} c_{\mathcal{F}}(\mathbf{s}, \cdot)$ commutes with the infimum operation for all $\mathbf{s} \in \mathcal{F}(\mathbf{X})$. In this case, the operator $\mathcal{E}_{\mathcal{F}}(\cdot, \mathbf{s})$ represents an erosion for every SE $\mathbf{s}$. Given a fuzzified set intersection $S e c_{\mathcal{F}}$ such that $\operatorname{Sec} c_{\mathcal{F}}(\mathbf{s}, \cdot)$ commutes with the supremum operation, we obtain a fuzzy dilation $\Delta_{\mathcal{F}}: \mathcal{F}(\mathbf{X}) \times \mathcal{F}(\mathbf{X}) \rightarrow \mathcal{F}(\mathbf{X})$ via the following definition (note that $\overline{\mathbf{s}}_{\mathbf{x}}(\mathbf{y})=\overline{\mathbf{s}}(\mathbf{y}-\mathbf{x})=\mathbf{s}(\mathbf{x}-\mathbf{y}) \forall \mathbf{y} \in \mathbf{X}$ ):

$\Delta_{\mathcal{F}}(\mathbf{a}, \mathbf{s})(\mathbf{x})=\operatorname{Sec}_{\mathcal{F}}\left(\overline{\mathbf{s}}_{\mathbf{x}}, \mathbf{a}\right)$.

Recall that almost all approaches towards fuzzy mathematical morphology employ inclusion measures based on infimums of fuzzy implications to generate fuzzy erosions as well as intersection measures based on supremums of fuzzy conjunctions to generate fuzzy dilations [80]. The following section generalizes this strategy to the $\mathbb{L}$-fuzzy setting.

\subsection{Some Basic Concepts and General Results on $\mathbb{L}$-fuzzy Mathematical Morphology}

Given $\mathbb{L}$-fuzzy inclusion and intersection measures, it is easy to construct operators $\mathcal{E}_{\mathbb{L}}, \Delta_{\mathbb{L}}: \mathcal{F}_{\mathbb{L}}(\mathbf{X}) \times \mathcal{F}_{\mathbb{L}}(\mathbf{X}) \rightarrow \mathcal{F}_{\mathbb{L}}(\mathbf{X})$ in analogy to equations 20 and 21 as follows:

$\mathcal{E}_{\mathbb{L}}(\mathbf{a}, \mathbf{s})(\mathbf{x})=\operatorname{In} c_{\mathbb{L}}\left(\mathbf{s}_{\mathbf{x}}, \mathbf{a}\right)$,

$\Delta_{\mathbb{L}}(\mathbf{a}, \mathbf{s})(\mathbf{x})=\operatorname{Sec}_{\mathbb{L}}\left(\overline{\mathbf{s}}_{\mathbf{x}}, \mathbf{a}\right)$.

In particular, if the $\mathbb{L}$-fuzzy inclusion and intersection measures occurring in Equations 22 and 23 are induced by an $\mathbb{L}$-fuzzy implication $I$ and an $\mathbb{L}$-fuzzy conjunction $C$ in terms of Equations 17 and 18. We obtain the following expressions for the operators $\mathcal{E}_{\mathbb{L}}$ and $\Delta_{\mathbb{L}}$ :

$$
\begin{aligned}
\mathcal{E}_{\mathbb{L}}(\mathbf{a}, \mathbf{s})(\mathbf{x}) & =\bigwedge_{\mathbf{y} \in \mathbf{X}} I\left(\mathbf{s}_{\mathbf{x}}(\mathbf{y}), \mathbf{a}(\mathbf{y})\right) \forall \mathbf{x} \in \mathbf{X} \\
\Delta_{\mathbb{L}}(\mathbf{a}, \mathbf{s})(\mathbf{x}) & =\bigvee_{\mathbf{y} \in \mathbf{X}} C\left(\overline{\mathbf{s}}_{\mathbf{x}}(\mathbf{y}), \mathbf{a}(\mathbf{y})\right) \forall \mathbf{x} \in \mathbf{X}
\end{aligned}
$$

Here, the $\mathbb{L}$-fuzzy set a plays the role of the image and the $\mathbb{L}$-fuzzy set $\mathbf{s}$ plays the role of the structuring element. We refer to the operator $\mathcal{E}_{\mathbb{L}}, \Delta_{\mathbb{L}}$ respectively, using the terminology $\mathbb{L}$-fuzzy erosion, $\mathbb{L}$-fuzzy dilation respectively, if $\mathcal{E}_{\mathbb{L}}(., \mathbf{s})$ represents an erosion in the sense of Equation 1 for every SE $\mathbf{s} \in \mathcal{F}_{\mathbb{L}}(\mathbf{X})$, if $\Delta_{\mathbb{L}}(., \mathbf{s})$ represents an dilation in the sense of Equation 1 for every $\mathrm{SE} \mathbf{s} \in \mathcal{F}_{\mathbb{L}}(\mathbf{X})$, respectively. In this case, we refer to $\mathcal{E}_{\mathbb{L}}(\mathbf{a}, \mathbf{s})$ as the $\mathbb{L}$-fuzzy erosion of the image a by the $S E \mathbf{s}$ and we refer to $\Delta_{\mathbb{L}}(\mathbf{a}, \mathbf{s})$ as the $\mathbb{L}-f u z z y$ dilation of the image a by the SE $\mathbf{s}$.

Theorem 5 Let the operator $\mathcal{E}_{\mathbb{L}}$ be induced by an Inf-I Inclusion measure,i.e., $\mathcal{E}_{\mathbb{L}}$ is given by Equations 22 and 24. The following statements are equivalent. 
1. The operators $\mathcal{E}_{\mathbb{L}}(., \mathbf{s})$ are erosions for all $\mathbf{s} \in \mathcal{F}_{\mathbb{L}}(\mathbf{X})$.

2. The operators $\operatorname{Inc}_{\mathbb{L}}(\mathbf{s},$.$) are erosions for all \mathbf{s} \in \mathcal{F}_{\mathbb{L}}(\mathbf{X})$.

3. The operators $I(s,$.$) are erosions for all s \in \mathbb{L}$.

The following, similar theorem concerns $\mathbb{L}$-fuzzy dilations.

Theorem 6 Let the operator $\Delta_{\mathbb{L}}$ be induced by a Sup-C intersection measure. The following statements are equivalent.

1. The operators $\Delta_{\mathbb{L}}(., \mathbf{s})$ are dilations for all $\mathbf{s} \in \mathcal{F}_{\mathbb{L}}(\mathbf{X})$.

2. The operators $\operatorname{Sec}_{\mathbb{L}}(\mathbf{s},$.$) are dilations for all \mathbf{s} \in \mathcal{F}_{\mathbb{L}}(\mathbf{X})$.

3. The operators $C(s,$.$) are dilations for all s \in \mathbb{L}$.

In MM, erosions and dilations usually occur in pairs whose constituents are dual to each other with respect to either adjunction or negation. Note that a negation $N$ on $\mathbb{L}$ induces a negation $\mathcal{N}$ on $\mathcal{F}_{\mathbb{L}}(\mathbf{X})$ by means of the equations $\mathcal{N}(\mathbf{a})(\mathbf{x})=N(\mathbf{a}(\mathbf{x}))$, where $\mathbf{a} \in \mathcal{F}_{\mathbb{L}}(\mathbf{X})$ and $\mathbf{x} \in \mathbf{X}$. For simplicity, we introduce the following nomenclatures.

Definition 6 Let $\mathcal{E}_{\mathbb{L}}$ and $\Delta_{\mathbb{L}}$ be $\mathcal{F}_{\mathbb{L}}(\mathbf{X}) \times \mathcal{F}_{\mathbb{L}}(\mathbf{X}) \rightarrow \mathcal{F}_{\mathbb{L}}(\mathbf{X})$ mappings. We say that the pair $\left(\mathcal{E}_{\mathbb{L}}, \Delta_{\mathbb{L}}\right)$ forms an adjunction if and only $\left(\mathcal{E}_{\mathbb{L}}(., \mathbf{s}), \Delta_{\mathbb{L}}(., \overline{\mathbf{s}})\right)$ forms an adjunction for every $\mathrm{SE} \mathbf{s} \in \mathcal{F}_{\mathbb{L}}(\mathbf{X})$. We say that $\mathcal{E}_{\mathbb{L}}$ and $\Delta_{\mathbb{L}}$ are dual with respect to a negation $N$ on $\mathbb{L}$ if and only if $\mathcal{E}_{\mathbb{L}}(., \mathbf{s})$ and $\Delta_{\mathbb{L}}(., \overline{\mathbf{s}})$ are dual with respect to the corresponding negation $\mathcal{N}$ on $\mathcal{F}_{\mathbb{L}}(\mathbf{X})$ for every SE $\mathbf{s} \in \mathcal{F}_{\mathbb{L}}(\mathbf{X})$.

Our focus is on operators $\mathcal{E}_{\mathbb{L}}$ and $\Delta_{\mathbb{L}}$ that are built from $\mathbb{L}$-fuzzy connectives by means of Equations 24 and 25 . The following theorem links a duality relationship between $\mathcal{E}_{\mathbb{L}}$ and $\Delta_{\mathbb{L}}$ to the corresponding duality relationship between the underlying $\mathbb{L}$-fuzzy connectives $I$ and $C$.

Theorem 7 Let I be an $\mathbb{L}$-fuzzy implication and $C$ be an $\mathbb{L}$-fuzzy conjunction. The pair $(I, C)$ forms an adjunction if and only if the corresponding pair $\left(\mathcal{E}_{\mathbb{L}}, \Delta_{\mathbb{L}}\right)$ given by Equations 24 and 25 forms an adjunction. Similarly, $I$ and $C$ are dual with respect to an $\mathbb{L}_{-}$-fuzzy negation $N$ if and only if the corresponding $\mathcal{F}_{\mathbb{L}}(\mathbf{X}) \times \mathcal{F}_{\mathbb{L}}(\mathbf{X}) \rightarrow \mathcal{F}_{\mathbb{L}}(\mathbf{X})$ mappings $\mathcal{E}_{\mathbb{L}}$ and $\Delta_{\mathbb{L}}$ are dual with respect to $N$.

For now, Theorem 7 concludes our investigation of theoretical aspects of general $\mathbb{L}$-fuzzy MM. The results of this section can be applied to any particular choice of $\mathbb{L}$ which are of practical interest, in particular to the classes of intervalvalued, intuitionistic, and bipolar fuzzy sets. Note that Theorem 7 refers to two possible approaches for constructing pairs consisting of an erosion and a dilation. In some recent papers, Isabelle Bloch has established links between these two approaches for the special cases of fuzzy sets and bipolar fuzzy sets $[12,13]$.

\section{Connectives for Interval-Valued and Intuitionistic Fuzzy Mathematical Morphologies}

4.1 Introduction and Basic Concepts of Interval-Valued and Intuitionistic Fuzzy Sets

In this paper, we are especially interested in interval-valued and intuitionistic fuzzy mathematical morphologies which we will treat as special cases of $\mathbb{L}$-fuzzy MM. This line of reasoning is made possible by the facts that the classes of interval-valued and intuitionistic fuzzy sets form complete lattices. In fact, these complete lattices are isomorphic [27]. Before going into details, let us briefly review intervalvalued and intuitionistic fuzzy sets.

As the name indicates, interval-valued fuzzy sets (IVFSs) on a universe $\mathbf{X}$ are mappings $A: \mathbf{X} \rightarrow \mathbb{L}^{I}$. Here, the symbol $\mathbb{L}^{I}$ denotes the set of all closed subintervals of $[0,1]$. An intuitionistic fuzzy set (IFS) is a mapping $A^{\prime}: \mathbf{X} \rightarrow \mathbb{L}^{*}$ where the symbol $\mathbb{L}^{*}$ denotes the set $\left\{(\mu, \nu) \in[0,1]^{2}\right.$ : $\mu+\nu \leq 1\}$. Both IVFSs and IFSs belong to the class of $\mathbb{L}$-fuzzy sets where $\mathbb{L}$ is a complete lattice $[27,30]$. The partial ordering on $[0,1]$ induces a partial ordering on the set $\mathbb{L}^{I}=\{[x, y] \subseteq[0,1]\}$ as follows:

$[u, v] \leq[x, y] \Leftrightarrow u \leq x$ and $v \leq y$.

If the symbol $0_{\mathbb{L}^{I}}$ denotes $\bigwedge \mathbb{L}^{I}$ and $1_{\mathbb{L}^{I}}$ denotes $\bigvee \mathbb{L}^{I}$ then we have $0_{\mathbb{L}^{I}}=[0,0]$ and $1_{\mathbb{L}^{I}}=[1,1]$. The complete lattice $\mathbb{L}^{*}=\{(x, y): x+y \leq 1\}$ is endowed with the following partial ordering:

$(u, v) \leq(x, y) \Leftrightarrow u \leq x$ and $v \geq y$.

The least element of $\mathbb{L}^{*}$, denoted by $0_{\mathbb{L}^{*}}$, is given by $(0,1)$ and the greatest element of $\mathbb{L}^{*}$, denoted by $0_{\mathbb{L}^{*}}$, is given by $(1,0)$. From now on, we will refer to the complete lattice of interval-valued fuzzy sets on $\mathbf{X}$ using the symbol $\mathbb{L}_{I V F S}$ and we will refer to the complete lattice of intuitionistic fuzzy sets on $\mathbf{X}$ using the symbol $\mathbb{L}_{I F S}$.

Several researchers have pointed out that the notions of interval-valued fuzzy sets and intuitionistic fuzzy sets are mathematically equivalent $[5,87]$. Most importantly, the complete lattices $\mathbb{L}_{I V F S}$ and $\mathbb{L}_{I F S}$ are isomorphic because the underlying complete lattices $\mathbb{L}^{I}$ and $\mathbb{L}^{*}$ are isomorphic [27, $30]$ in terms of $\phi$ given as follows:

$$
\begin{aligned}
\phi: \mathbb{L}^{I} & \rightarrow \mathbb{L}^{*} \\
{[x, y] } & \mapsto(x, 1-y)
\end{aligned}
$$

The lattice isomorphism $\phi$ induces a lattice isomorphism $\Phi: \mathbb{L}_{I V F S} \rightarrow \mathbb{L}_{I F S}$ that maps an IVFS $A$ with $A(\mathbf{x})=$ $[\underline{A}(\mathbf{x}), \bar{A}(\mathbf{x})]$ for all $\mathbf{x} \in \mathbf{X}$ to the IFS $A^{\prime}$ that satisfies $A^{\prime}(\mathbf{x})=(\underline{A}(\mathbf{x}), 1-\bar{A}(\mathbf{x}))$. The inverse of $\phi$ associates an element $(\mu, \nu) \in \mathbb{L}^{*}$ with $[\mu, 1-\nu] \in \mathbb{L}^{I}$ and induces the lattice isomorphism $\Phi^{-1}$ from $\mathbb{L}_{I F S}$ to $\mathbb{L}_{I V F S}$. 
Note that elements of $[0,1]$ can be identified with elements of $\mathbb{L}^{I}$ of the form $[x, x]$ and with elements of $\mathbb{L}^{*}$ of the form $(x, 1-x)=\phi([x, x])$. In other words, the unit interval $[0,1]$ can be considered a subset of $\mathbb{L}^{I}$ as well as of $\mathbb{L}^{*}$. Therefore, the class of fuzzy sets $\mathcal{F}(\mathbf{X})$ over the universe $\mathbf{X}$ is contained in the class of interval-valued fuzzy sets $\mathbb{L}_{I V F S}$ as well as in the class of intuitionistic fuzzy sets $\mathbb{L}_{\text {IFS }}$ over $\mathbf{X}$.

In the remainder of this section, we focus our attention on operations on the complete lattice $\mathbb{L}_{I F S}$, citing some specific examples of intuitionistic fuzzy operations that have appeared in the literature. Generalizations of the Gödel implication $I_{M}$ are given by the following intuitionistic implications $\mathcal{I}_{M}^{*}, \mathcal{I}_{A G}^{*}$, and $\mathcal{I}_{S}^{*}[19,6,18]$ :

$\mathcal{I}_{M}^{*}(\mathbf{u}, \mathbf{v})= \begin{cases}1_{\mathbb{L}^{*}} & \text { if } \mathbf{u} \leq \mathbf{v} \\ \mathbf{v} & \text { otherwise }\end{cases}$

$\mathcal{I}_{A G}^{*}(\mathbf{u}, \mathbf{v})= \begin{cases}1_{\mathbb{L}^{*}} & \text { if } u_{1} \leq v_{1}, \\ \left(v_{1}, 0\right) & \text { if } u_{1}>v_{1} \text { and } u_{2} \geq v_{2}, \\ \left(v_{1}, v_{2}\right) & \text { if } u_{1}>v_{1} \text { and } u_{2}<v_{2} .\end{cases}$

$\mathcal{I}_{S}^{*}(\mathbf{u}, \mathbf{v})= \begin{cases}1_{\mathbb{L}^{*}} & \text { if } \mathbf{u} \leq \mathbf{v}, \\ \left(1-v_{2}, v_{2}\right) & \text { if } u_{1} \leq v_{1} \text { and } u_{2}<v_{2} \\ \left(v_{1}, 0\right) & \text { if } u_{1}>v_{1} \text { and } u_{2} \geq v_{2} \\ \mathbf{v} & \text { if } u_{1}>v_{1} \text { and } u_{2}<v_{2}\end{cases}$

In contrast to intuitionistic and interval-valued implications, general intuitionistic or interval-valued fuzzy conjunctions have not been extensively studied in the literature. Instead, researchers have concentrated on the special classes of intuitionistic and interval-valued $t$-norms [25, 26, 29]. Examples of intuitionistic $t$-norms include the following $[4,18$, 26, 29]:

$$
\begin{aligned}
\mathcal{T}_{M}^{*}(\mathbf{u}, \mathbf{v})= & \mathbf{u} \wedge \mathbf{v}=\left(u_{1} \wedge v_{1}, u_{2} \vee v_{2}\right), \\
\mathcal{T}_{W}^{*}(\mathbf{u}, \mathbf{v})= & \left(0 \vee\left(u_{1}+v_{1}-1\right), 1 \wedge\left(u_{2}+v_{2}\right)\right), \\
\mathcal{T}_{A}^{*}(\mathbf{u}, \mathbf{v})= & \left(u_{1} v_{1}, u_{2}+v_{2}-u_{2} v_{2}\right), \\
\mathcal{T}_{L}^{*}(\mathbf{u}, \mathbf{v})= & \left(0 \vee\left(u_{1}+v_{1}-1\right),\right. \\
& \left.1 \wedge\left(u_{2}+1-v_{1}\right) \wedge\left(v_{2}+1-u_{1}\right)\right) .
\end{aligned}
$$

As far as the definition of a negation on $\mathbb{L}^{I}$ or $\mathbb{L}^{*}$ is concerned, several researchers have left away the involutiveness requirement $[18,26,30]$. Thus, these definitions neither comply with the definition of a negation in the complete lattice framework of mathematical morphology [37] nor with the types of negations that have been used by researchers in fuzzy mathematical morphology $[15,56,70,80]$. Since we view interval-valued and intuitionistic FMMs both as special cases of $\mathbb{L}$-fuzzy MM and as extensions of FMM, we additionally require negations on $\mathbb{L}^{*}$ and on $\mathbb{L}^{I}$ to be involutive in accordance with our definition of negation in Section 2.1. Deschrijver, Cornelis, and Kerre have completely characterized (involutive) negations on $\mathbb{L}^{*}$ and on $\mathbb{L}^{I}[25,26]$. In particular, the standard negations $\mathcal{N}_{S}^{*}$ on $\mathbb{L}^{*}$ and $\mathcal{N}_{S}$ on $\mathbb{L}^{I}$ are given as follows:

$$
\begin{aligned}
& \mathcal{N}_{S}^{*}(\mathbf{u})=\left(u_{2}, u_{1}\right) \forall \mathbf{u} \in \mathbb{L}^{*}, \\
& \mathcal{N}_{S}(\mathbf{x})=\left[1-x_{2}, 1-x_{1}\right] \forall \mathbf{x} \in \mathbb{L}^{I} .
\end{aligned}
$$

Note that, given an implication $\mathcal{I}^{*}$, a conjunction $\mathcal{C}^{*}$, and a negation $\mathcal{N}^{*}$ on $\mathbb{L}^{*}$, we are able to construct a specific approach to intuitionistic FMM. Intuitionistic fuzzy inclusion and intersection measures $I n c_{\mathbb{L}} *$ and $S e c_{\mathbb{L}} *$ immediately arise as the inf- $\mathcal{I}^{*}$ inclusion measure and the sup- $\mathcal{C}^{*}$ intersection measure defined in Equations 17 and 18. Then, Equations 24 and 25 lead to intuitionistic fuzzy operators $\mathcal{E}_{\mathbb{L}^{*}}$ and $\Delta_{\mathbb{L}^{*}}$. Theorem 7 implies that $\mathcal{I}^{*}$ and $\mathcal{C}^{*}$ are dual with respect to $\mathcal{N}^{*}$ if and only if $\mathcal{E}_{\mathbb{L}^{*}}$ and $\Delta_{\mathbb{L}^{*}}$ are dual with respect to $\mathcal{N}^{*}$. Moreover, $\mathcal{I}^{*}$ and $\mathcal{C}^{*}$ are adjoint if and only if $\mathcal{E}_{\mathbb{L}^{*}}$ and $\Delta_{\mathbb{L}^{*}}$ are adjoint. Similar remarks can be made in the interval-valued fuzzy case.

\subsection{Construction of Interval-Valued and Intuitionistic Fuzzy Operators}

The development of various approaches to interval-valued or intuitionistic FMM based on interval-valued or intuitionistic Inf-I inclusion and Sup-C intersection measures presumes the availability of interval-valued or intuitionistic implications and conjunctions. Ideally, researchers and practitioners have a variety of interval-valued and intuitionistic implications and conjunctions at their disposal including criteria that facilitate the choice of a particular approach to intervalvalued or intuitionistic FMM that is suited for a given application [57-59]. As a first step in this direction, this section presents several strategies for constructing new intervalvalued and intuitionistic fuzzy operators.

\subsubsection{Operators Derived from the Lattice Isomorphism between $\mathbb{L}^{I}$ and $\mathbb{L}^{*}$}

The following theorems reveal that there is a natural oneto-one correspondence between operators on $\mathbb{L}^{*}$ and operators on $\mathbb{L}^{I}$ that allows for the construction of interval-valued fuzzy connectives from intuitionistic fuzzy connectives and vice-versa. Recall that $\phi$ denotes the lattice isomorphism $\mathbb{L}^{I} \rightarrow \mathbb{L}^{*}$ that was defined in Equation 28.

Theorem 8 Consider the following mapping $\mathcal{M}$ that associates functions $\mathcal{F}^{*}:\left(\mathbb{L}^{*}\right)^{2} \rightarrow \mathbb{L}^{*}$ with functions $\mathcal{F}=$ 
$\mathcal{M}\left(\mathcal{F}^{*}\right):\left(\mathbb{L}^{I}\right)^{2} \rightarrow \mathbb{L}^{I}$ where $\mathcal{F}=\mathcal{M}\left(\mathcal{F}^{*}\right)$ is defined as follows for all $\mathbf{x}, \mathbf{y} \in \mathbb{L}^{I}$.

$\mathcal{F}(\mathbf{x}, \mathbf{y})=\phi^{-1}\left(\mathcal{F}^{*}(\phi(\mathbf{x}), \phi(\mathbf{y}))\right)$.

The inverse of $\mathcal{M}$ is given by

$$
\mathcal{M}^{-1}(\mathcal{F})(\mathbf{u}, \mathbf{v})=\mathcal{F}^{*}(\mathbf{u}, \mathbf{v})=\phi\left(\mathcal{F}\left(\phi^{-1}(\mathbf{u}), \phi^{-1}(\mathbf{v})\right)\right) .
$$

Moreover, the following statements are satisfied.

1. The operator $\mathcal{F}^{*}$ is an implication on $\mathbb{L}^{*}$ if and only if $\mathcal{F}$ is an implication on $\mathbb{L}^{I}$. The corresponding statements also hold true for conjunctions and disjunctions.

2. The operator $\mathcal{F}^{*}$ is a t-norm on $\mathbb{L}^{*}$ if and only if $\mathcal{F}$ is a t-norm on $\mathbb{L}^{I}$. The operator $\mathcal{F}^{*}$ is an s-norm on $\mathbb{L}^{*}$ if and only if $\mathcal{F}$ is an s-norm on $\mathbb{L}^{I}$.

3. An implication $\mathcal{I}^{*}$ on $\mathbb{L}^{*}$ and a conjunction $\mathcal{C}^{*}$ on $\mathbb{L}^{*}$ are adjoint if and only if the respective implication $\mathcal{I}=$ $\mathcal{M}\left(\mathcal{I}^{*}\right)$ on $\mathbb{L}^{I}$ and the respective conjunction $\mathcal{C}=\mathcal{M}\left(\mathcal{C}^{*}\right)$ on $\mathbb{L}^{I}$ are adjoint.

In Equations 29 to 31, we presented three intuitionistic fuzzy extensions of the Gödel implication $I_{M}$. An application of Theorem 8 yields three interval-valued fuzzy implications that extend the Gödel implication $I_{M}$. First, note that

$\mathcal{I}_{M}^{*}(\phi(\mathbf{x}), \phi(\mathbf{y}))= \begin{cases}1_{\mathbb{L}^{*}} & \text { if } \phi(\mathbf{x}) \leq \phi(\mathbf{y}) \Leftrightarrow \mathbf{x} \leq \mathbf{y} \\ \phi(\mathbf{y}) & \text { otherwise }\end{cases}$

Therefore, $\mathcal{I}_{M}^{*}$ corresponds to the following implication $\mathcal{I}_{M}$ on $\mathbb{L}^{I}$ :

$\mathcal{I}_{M}(\mathbf{x}, \mathbf{y})= \begin{cases}1_{\mathbb{L}^{I}} & \text { if } \mathbf{x} \leq \mathbf{y} \\ \mathbf{y} & \text { otherwise }\end{cases}$

In a similar way, we derive the interval-valued implication $\mathcal{I}_{A G}$ corresponding to Atanassov's and Gargov's intuitionistic implication and the interval-valued implication $\mathcal{I}_{S}$ corresponding to the intuitionistic implication $\mathcal{I}_{S}^{*}$. We obtain:

$$
\begin{aligned}
& \mathcal{I}_{A G}(\mathbf{x}, \mathbf{y})= \begin{cases}1_{\mathbb{L}^{I}} & \text { if } x_{1} \leq y_{1}, \\
{\left[y_{1}, 1\right]} & \text { if } x_{1}>y_{1} \text { and } x_{2} \leq y_{2}, \\
\mathbf{y} & \text { if } \mathbf{x} \geq \mathbf{y}\end{cases} \\
& \mathcal{I}_{S}(\mathbf{x}, \mathbf{y})= \begin{cases}1_{\mathbb{L}^{I}} & \text { if } \mathbf{x} \leq \mathbf{y}, \\
{\left[y_{2}, y_{2}\right]} & \text { if } x_{1} \leq y_{1} \text { and } x_{2}>y_{2}, \\
{\left[y_{1}, 1\right]} & \text { if } x_{1}>y_{1} \text { and } x_{2} \leq y_{2}, \\
\mathbf{y} & \text { if } \mathbf{x}>\mathbf{y} .\end{cases}
\end{aligned}
$$

An application of Theorem 8 to the intuitionistic fuzzy $t$-norms listed in Equations 32 to 35 yields the following interval-valued $t$-norms $\mathcal{T}_{M}, \mathcal{T}_{W}, \mathcal{T}_{A}$, and $\mathcal{T}_{L}$ :

$$
\begin{aligned}
\mathcal{T}_{M}(\mathbf{x}, \mathbf{y})= & \mathbf{x} \wedge \mathbf{y}=\left[x_{1} \wedge y_{1}, x_{2} \wedge y_{2}\right] \\
\mathcal{T}_{W}(\mathbf{x}, \mathbf{y})= & {\left[0 \vee\left(x_{1}+y_{1}-1\right), 0 \vee\left(x_{2}+y_{2}-1\right)\right], } \\
\mathcal{T}_{A}(\mathbf{x}, \mathbf{y})= & {\left[x_{1} y_{1}, x_{2} y_{2}\right] } \\
\mathcal{T}_{L}(\mathbf{x}, \mathbf{y})= & {\left[0 \vee\left(x_{1}+y_{1}-1\right),\right.} \\
& \left.0 \vee\left(x_{2}+y_{1}-1\right) \vee\left(y_{2}+x_{1}-1\right)\right]
\end{aligned}
$$

The fact that $[0,1]$ constitutes an infinitely distributive lattice [9] implies that the intuitionistic $t$-norm $\mathcal{T}_{M}^{*}$ is a dilation in both arguments. Therefore, the adjoint erosion on $\mathbb{L}^{*}$ can be constructed by using Proposition 1 . This procedure gives rise to the $R$-implicator $\mathcal{I}_{M}^{*}$ of $\mathcal{T}_{M}^{*}$ on $\mathbb{L}^{*}$. By Theorem 1, the R-implicator $\mathcal{I}_{\mathcal{T}}^{*}$ of an intuitionistic $t$-norm $\mathcal{T}^{*}$ can be computed as follows:

$\mathcal{I}_{\mathcal{T}}^{*}(\mathbf{u}, \mathbf{w})=\bigvee\left\{\mathbf{v} \in \mathbb{L}^{*}: \mathcal{T}^{*}(\mathbf{u}, \mathbf{v}) \leq \mathbf{w}\right\} \forall \mathbf{u}, \mathbf{w} \in \mathbb{L}^{*}$

Since Cornelis et al. have shown that the R-implicator of $\mathcal{T}_{M}^{*}$, where $\mathcal{T}_{M}^{*}(\mathbf{u}, \mathbf{v})=\mathbf{u} \wedge \mathbf{v}$ for all $\mathbf{u}, \mathbf{v} \in \mathbb{L}^{*}$, is given by the intuitionistic fuzzy implication $\mathcal{I}_{S}^{*}$ of Equation 31 [18], we have that $\mathcal{I}_{S}^{*}$ and $\mathcal{T}_{M}^{*}$ are adjoint. Therefore, Part 3 of Theorem 8 implies that the interval-valued implication $\mathcal{I}_{S}$ and the interval-valued $t$-norm $\mathcal{T}_{M}$ are adjoint as well. Finally note that the intuitionistic maximum $\mathcal{S}_{M}^{*}$ corresponds to the interval-valued maximum $\mathcal{S}_{M}$, where $\mathcal{S}_{M}(\mathbf{x}, \mathbf{y})=$ $\mathbf{x} \vee \mathbf{y}$.

Theorem 9 The following mapping $\mathcal{P}$ defines a bijection between the set of negations on $\mathbb{L}^{*}$ and the set of negations on $\mathbb{L}^{I}$. If $\mathcal{N}^{*}$ is an intuitionistic fuzzy negation then an interval-valued fuzzy negation $\mathcal{N}=\mathcal{P}\left(\mathcal{N}^{*}\right)$ is given as follows.

$\mathcal{N}(\mathbf{x})=\phi^{-1}\left(\mathcal{N}^{*}(\phi(\mathbf{x}))\right), \forall \mathbf{x} \in \mathbb{L}^{I}$

The inverse of $\mathcal{P}$ associates an interval-valued fuzzy negation $\mathcal{N}$ with an intuitionistic fuzzy negation $\mathcal{N}^{*}=\mathcal{P}^{-1}(\mathcal{N})$ which can be computed as follows.

$\mathcal{N}^{*}(\mathbf{u})=\phi\left(\mathcal{N}\left(\phi^{-1}(\mathbf{u})\right)\right), \forall \mathbf{u} \in \mathbb{L}^{*}$.

A brief glance at Equations 36 and 37 reveals that the bijection $\mathcal{P}$ maps $\mathcal{N}_{S}$ to $\mathcal{N}_{S}^{*}$.

Theorem 10 Let $\mathcal{M}$ and $\mathcal{P}$ be as in Theorems 8 and 9. Two intuitionistic fuzzy connectives $\mathcal{F}^{*}$ and $\mathcal{G}^{*}$ are dual with respect to an intuitionistic fuzzy negation $\mathcal{N}^{*}$ in the sense 
of Definition 2 if and only if the corresponding intervalvalued fuzzy connectives $\mathcal{F}=\mathcal{M}\left(\mathcal{F}^{*}\right)$ and $\mathcal{G}=\mathcal{M}\left(\mathcal{G}^{*}\right)$ are dual with respect to the interval-valued fuzzy negation $\mathcal{N}=\mathcal{P}\left(\mathcal{N}^{*}\right)$.

The following equalities reveal that the intuitionistic minimum $\mathcal{T}_{M}^{*}$ and the intuitionistic maximum $\mathcal{S}_{M}^{*}$ are dual with respect to the standard intuitionistic fuzzy negator $\mathcal{N}_{S}^{*}$.

$$
\begin{aligned}
& \mathcal{N}_{S}^{*}\left(\mathcal{S}_{M}^{*}\left(\mathcal{N}_{S}^{*}(\mathbf{u}), \mathcal{N}_{S}^{*}(\mathbf{v})\right)\right)=\mathcal{N}_{S}^{*}\left(\mathcal{N}_{S}^{*}(\mathbf{u}) \vee \mathcal{N}_{S}^{*}(\mathbf{v})\right) \\
& =\mathcal{N}_{S}^{*}\left(\left(u_{2}, u_{1}\right) \vee\left(v_{2}, v_{1}\right)\right)=\mathcal{N}_{S}^{*}\left(\left(u_{2} \vee v_{2}, u_{1} \wedge v_{1}\right)\right) \\
& =\left(u_{1} \wedge v_{1}, u_{2} \vee v_{2}\right)=\mathbf{u} \wedge \mathbf{v}=\mathcal{T}_{M}^{*}(\mathbf{u}, \mathbf{v}) .
\end{aligned}
$$

Thus, by Theorem 10, the interval-valued minimum $\mathcal{T}_{M}=$ $\mathcal{M}\left(\mathcal{T}_{M}^{*}\right)$ and the interval-valued maximum $\mathcal{S}_{M}=\mathcal{M}\left(\mathcal{S}_{M}^{*}\right)$ are dual with respect to the standard interval-valued negator $\mathcal{N}_{S}=\mathcal{P}\left(\mathcal{N}_{S}^{*}\right)$. The following extension $\mathcal{I}_{\mathcal{S}_{M}^{*}, \mathcal{N}_{S}^{*}}^{*}$ of the Kleene-Deenes implicator $I_{K}$ has been shown to be the dual of the intuitionistic maximum operator $\mathcal{S}_{M}^{*}$ with respect to the standard intuitionistic negation $\mathcal{N}_{S}^{*}[4,6,18]$.

$\mathcal{I}_{\mathcal{S}_{M}^{*}, \mathcal{N}_{S}^{*}}^{*}(\mathbf{u}, \mathbf{v})=\left(u_{2} \vee v_{1}, u_{1} \wedge v_{2}\right)$.

By Theorem 10, forming the image of $\mathcal{I}_{\mathcal{S}_{M}^{*}, \mathcal{N}_{S}^{*}}^{*}$ under the bijection $\mathcal{M}$ produces the interval-valued fuzzy implication $\mathcal{I}_{\mathcal{S}_{M}, \mathcal{N}_{S}}$ that is the dual of the interval-valued maximum operator $\mathcal{S}_{M}$ with respect to the standard interval-valued negation $\mathcal{N}_{S}$. Thus, we obtain

$$
\begin{aligned}
& \mathcal{I}_{\mathcal{S}_{M}, \mathcal{N}_{S}}(\mathbf{x}, \mathbf{y})=\mathcal{M}\left(\mathcal{I}_{\mathcal{S}_{M}, \mathcal{N}_{S}}\right)(\mathbf{x}, \mathbf{y}) \\
& =\phi^{-1}\left(\mathcal{I}_{\mathcal{S}_{M}, \mathcal{N}_{S}^{*}}^{*}(\phi(\mathbf{x}), \phi(\mathbf{y}))\right) \\
& =\phi^{-1}\left(\mathcal{I}_{\mathcal{S}_{M}, \mathcal{N}_{S}^{*}}^{*}\left(\left(x_{1}, 1-x_{2}\right),\left(y_{1}, 1-y_{2}\right)\right)\right) \\
& =\phi^{-1}\left(\left(1-x_{2}\right) \vee y_{1}, x_{1} \wedge\left(1-y_{2}\right)\right) \\
& =\left[\left(1-x_{2}\right) \vee y_{1},\left(1-x_{1}\right) \vee y_{2}\right] .
\end{aligned}
$$

\subsubsection{Interval-Valued and Intuitionistic Operators Based on Fuzzy Operators}

As we will point out in this section, a large class of intuitionistic and interval-valued fuzzy connectives can be constructed from fuzzy logical connectives on $[0,1]$. For simplicity, we focus on interval-valued fuzzy connectives. Their intuitionistic fuzzy counterparts can be obtained via Equation 39. In particular, the following definitions of $t$-representable operators on $\mathbb{L}^{I}$ can be related in terms of Theorem 8 to the respective definitions of $t$-representable operators on $\mathbb{L}^{*}[17,18]$.

Definition 7 A conjunction $\mathcal{C}$ on $\mathbb{L}^{I}$ is called $C$-representable if $\mathcal{C}$ equals $\mathcal{C}_{C, C^{\prime}}^{r}$ for some fuzzy conjunctions $C$ and $C^{\prime}$ where $\mathcal{C}_{C, C^{\prime}}^{r}$ is defined as follows:
$\mathcal{C}_{C, C^{\prime}}^{r}(\mathbf{x}, \mathbf{y})=\left[C\left(x_{1}, y_{1}\right), C^{\prime}\left(x_{2}, y_{2}\right)\right]$

A disjunction $\mathcal{D}$ on $\mathbb{L}^{I}$ is called $C$-representable if $\mathcal{D}$ equals $\mathcal{D}_{D, D^{\prime}}^{r}$ for some fuzzy disjunctions $D$ and $D^{\prime}$ where $\mathcal{D}_{D, D^{\prime}}$ is defined as follows:

$\mathcal{D}_{D, D^{\prime}}^{r}(\mathbf{x}, \mathbf{y})=\left[D\left(x_{1}, y_{1}\right), D^{\prime}\left(x_{2}, y_{2}\right)\right]$

If both $C$ and $C^{\prime}$ are $t$-norms then we refer to $\mathcal{C}$ as $t$ representable. Similarly, if both $D$ and $D^{\prime}$ are $s$-norms then we refer to $\mathcal{D}$ as $t$-representable.

Obviously, $t$-representable conjunctions and disjunctions on $\mathbb{L}^{I}$ constitute respectively $t$-norms and $s$-norms on $\mathbb{L}^{I}$. Let us proceed by stating conditions that yield $C$-representable conjunctions and disjunctions on $\mathbb{L}^{I}$.

Theorem 11 The function $\mathcal{C}_{C, C^{\prime}}^{r}$ defined in Equation 54 represents a conjunction on $\mathbb{L}^{I}$ if and only if $C(x, y) \leq C^{\prime}(x, y)$ for all $x, y \in[0,1]$. Moreover, the function $\mathcal{D}_{D, D^{\prime}}^{r}$ defined in Equation 55 represents a disjunction on $\mathbb{L}^{I}$ if and only if $D(x, y) \leq D^{\prime}(x, y)$ for all $x, y \in[0,1]$.

The conditions of Theorem 11 are trivially satisfied if $C=C^{\prime}$ (or if $D=D^{\prime}$ ). Thus, the Łukasiewicz $t$-norm $T_{W}$ gives rise to the interval-valued $t$-norm $\mathcal{T}_{W}^{r}=\mathcal{T}_{W}$ of Equation 45 and the conjunction of Kleene and Dienes, denoted by $C_{K}$, gives rise to the following interval-valued fuzzy conjunction $\mathcal{C}_{K}^{r}$ :

$$
\begin{aligned}
\mathcal{C}_{K}^{r}(\mathbf{x}, \mathbf{y})= & {\left[C_{K}\left(x_{1}, y_{1}\right), C_{K}\left(x_{2}, y_{2}\right)\right] } \\
= & {\left[x_{1} \wedge H_{0}\left(x_{1}+y_{1}-1\right),\right.} \\
& \left.x_{2} \wedge H_{0}\left(x_{2}+y_{2}-1\right)\right]
\end{aligned}
$$

where

$H_{0}(x)=\left\{\begin{array}{l}0, x \leq 0, \\ 1, x>0 .\end{array} \quad \forall x \in \mathbb{R}\right.$.

It is a well-known fact that the minimum $t$-norm $T_{M}$ represents the pointwise largest $t$-norm [61]. Therefore, Theorem 11 yields the following $t$-norm $\mathcal{T}_{P, M}^{r}$ and the following conjunction $\mathcal{C}_{K, M}^{r}$ on $\mathbb{L}^{I}$.

$$
\begin{aligned}
\mathcal{T}_{P, M}^{r}(\mathbf{x}, \mathbf{y}) & =\left[T_{P}\left(x_{1}, y_{1}\right), T_{M}\left(x_{2}, y_{2}\right)\right] \\
& =\left[x_{1} \cdot y_{1}, x_{2} \wedge y_{2}\right] \\
\mathcal{C}_{K, M}^{r}(\mathbf{x}, \mathbf{y}) & =\left[C_{K}\left(x_{1}, y_{1}\right), T_{M}\left(x_{2}, y_{2}\right)\right] \\
& =\left[x_{1} \wedge H_{0}\left(x_{1}+y_{1}-1\right), x_{2} \wedge y_{2}\right]
\end{aligned}
$$

Definition 7 reveals that $C$-representable fuzzy operators on $\mathbb{L}^{I}$ can be generated by applying conventional fuzzy operators to the lower and upper bounds of the considered 
intervals. Definition 7 can be modified by lowering the upper bound (pessimistic approach) or by lifting the lower bound (optimistic approach). Generalizing previous definitions of pseudo- $t$-representable operators [25], we define pseudo- $C$ representable operators as follows. Here, we refrain from using two representatives since otherwise we would have several possible definitions of pessimistic operators and optimistic operators to choose from.

Definition 8 Let $C$ be a fuzzy conjunction. The pessimistic conjunction $\mathcal{C}_{C}^{p}$ and the optimistic conjunction $\mathcal{C}_{C}^{o}$ with representative $C$ is defined as follows:

$\mathcal{C}_{C}^{p}(\mathbf{x}, \mathbf{y})=\left[C\left(x_{1}, y_{1}\right), C\left(x_{1}, y_{2}\right) \vee C\left(x_{2}, y_{1}\right)\right]$,

$\mathcal{C}_{C}^{o}(\mathbf{x}, \mathbf{y})=\left[C\left(x_{1}, y_{2}\right) \wedge C\left(x_{2}, y_{1}\right), C\left(x_{2}, y_{2}\right)\right]$.

Likewise, if $D$ is fuzzy disjunction then the pessimistic disjunction $\mathcal{D}_{D}^{p}$ and the optimistic conjunction $\mathcal{D}_{D}^{o}$ with representative $D$ are defined as follows:

$\mathcal{D}_{D}^{p}(\mathbf{x}, \mathbf{y})=\left[D\left(x_{1}, y_{1}\right), D\left(x_{1}, y_{2}\right) \vee D\left(x_{2}, y_{1}\right)\right]$,

$\mathcal{D}_{D}^{o}(\mathbf{x}, \mathbf{y})=\left[D\left(x_{1}, y_{2}\right) \wedge D\left(x_{2}, y_{1}\right), D\left(x_{2}, y_{2}\right)\right]$.

As the reader may have already perceived, the intervalvalued conjunction $\mathcal{T}_{L}$ of Equation 47 is given by the pessimistic approach in conjunction with the representative $T_{W}$. As we have seen, numerous types of $C$-representable and pseudo- $C$-representable conjunctions and disjunctions can be constructed by means of Definitions 7 and 8 . In a similar vein, a large variety of $I$-representable and pseudo- $I$ representable implications arise from the following definitions [25].

Definition 9 Let $I$ and $I^{\prime}$ be fuzzy implications such that $I(x, y) \leq I^{\prime}(x, y)$ for all $x, y \in[0,1]$. We define the $I$ representable implication $\mathcal{I}_{I, I^{\prime}}^{r}$ with representatives $I$ and $I^{\prime}$, the pessimistic implication $\mathcal{I}_{I}^{p}$ with representative $I$, and the optimistic implication $\mathcal{I}_{I}^{o}$ with representative $I$ as follows:

$$
\begin{aligned}
& \mathcal{I}_{I, I^{\prime}}^{r}(\mathbf{x}, \mathbf{y})=\left[I\left(x_{2}, y_{1}\right), I^{\prime}\left(x_{1}, y_{2}\right)\right], \\
& \mathcal{I}_{I}^{p}(\mathbf{x}, \mathbf{y})=\left[I\left(x_{2}, y_{1}\right), I\left(x_{1}, y_{1}\right) \vee I\left(x_{2}, y_{2}\right)\right], \\
& \mathcal{I}_{I}^{o}(\mathbf{x}, \mathbf{y})=\left[I\left(x_{1}, y_{1}\right) \wedge I\left(x_{2}, y_{2}\right), I\left(x_{1}, y_{2}\right)\right] .
\end{aligned}
$$

For example, the Gödel implication $I_{M}$ and the Goguen implication $I_{P}$ satisfy the inequality $I_{M}(x, y) \leq I_{P}(x, y)$ for all $x, y \in[0,1]$ and allow for the construction of the $I$-representable implication with representatives $I_{M}$ and $I_{P}$ as well as the pessimistic and optimistic implications with either one of the representatives $I_{M}$ or $I_{P}$. For simplicity, we denote these implications using the symbols $\mathcal{I}_{M, P}^{r}, \mathcal{I}_{M}^{p}$, $\mathcal{I}_{P}^{p}, \mathcal{I}_{M}^{o}$, and $\mathcal{I}_{P}^{o}$.

Clearly, representable and pseudo-representable implications and conjunctions on $\mathbb{L}^{I}$ (on $\mathbb{L}^{*}$, respectively) can be inserted in Equations 22 and 23 in order to generate intervalvalued (intuitionistic) fuzzy operators $\mathcal{E}_{\mathcal{I}}$ and $\Delta_{\mathcal{C}}$ that form the basis of a particular approach to interval-valued (intuitionistic) FMM. If the operators $\mathcal{E}_{\mathcal{I}}$ and $\Delta_{\mathcal{C}}$ form an adjunction then their compositions yield (algebraic) openings and closings which in turn can be used to construct a variety of morphological filters [37]. On one hand, if $\mathcal{E}_{\mathcal{I}}$ and $\Delta_{\mathcal{C}}$ are adjoint then $\mathcal{E}_{\mathcal{I}}(., \mathbf{s})$ is necessarily an erosion and $\Delta_{\mathcal{C}}(., \mathbf{s})$ is necessarily a dilation for all structuring elements $\mathbf{s} \in \mathbb{L}_{I V F S}$ or, equivalently, $\mathcal{I}(\mathbf{z},$.$) is an erosion and \mathcal{C}(\mathbf{z},$. is a dilation for all $\mathbf{z} \in \mathbb{L}^{I}$. On the other hand, given an implication $\mathcal{I}$ on $\mathbb{L}^{I}$ such that $\mathcal{I}(\mathbf{z},$.$) are erosions for all$ $\mathbf{z} \in \mathbb{L}^{I}$, we can easily construct the adjoint conjunction $\mathcal{C}$ on $\mathbb{L}^{I}$ using Equation 12 . Conversely, given a conjunction $\mathcal{C}$ on $\mathbb{L}^{I}$ such that $\mathcal{C}(\mathbf{z},$.$) are dilations for all \mathbf{z} \in \mathbb{L}^{I}$, one can construct the adjoint implication using Equation 10. Both schemes produce an adjunction $\left(\mathcal{E}_{\mathcal{I}}, \Delta_{\mathcal{C}}\right)$.

By the following theorem, representable and pseudo-representable interval-valued connectives that are erosive or dilative in the second argument arise naturally from fuzzy connectives having the same property.

Theorem 12 If $C, C^{\prime}$ and $I, I^{\prime}$ are respectively fuzzy conjunctions and fuzzy implications such that $C(x, y) \leq C^{\prime}(x, y)$ and $I(x, y) \leq I^{\prime}(x, y)$ for all $x, y \in[0,1]$ then the following statements hold true:

1. The interval-valued conjunctions $\mathcal{C}_{C, C^{\prime}}^{r}$ and $\mathcal{C}_{C}^{p}$ represent dilations in the second component for every fixed first component $\mathbf{z} \in \mathbb{L}^{I}$ if and only if the conjunctions $C$ and $C^{\prime}$ represent dilations in the second component for every fixed first component $z \in[0,1]$.

2. The interval-valued implications $\mathcal{I}_{I, I^{\prime}}^{r}$ and $\mathcal{I}_{I}^{o}$ represent erosions in the second component for every fixed first component $\mathbf{z} \in \mathbb{L}^{I}$ if and only if the implications $I$ and $I^{\prime}$ represent erosions in the second component for every fixed first component $z \in[0,1]$.

For example, the interval-valued $t$-norms $\mathcal{T}_{M}, \mathcal{T}_{W}$, and $\mathcal{T}_{A}$ are all dilative in the second argument because they are respectively $t$-representable in terms of the minimum, the Łukasiewicz, and the product $t$-norms, which are dilative in the second argument. Another example of an interval-valued $t$-norm that is dilative in the second argument is given by $\mathcal{T}_{L}$ of Equation 47 since $\mathcal{T}_{L}$ equals $\mathcal{T}_{W}^{p}$, i.e., the pessimistic conjunction with the single representative $T_{W}$.

The optimistic conjunction $\mathcal{C}_{C}^{o}$ does generally not yield a dilation for fixed $\mathbf{z} \in \mathbb{L}^{I}$ even if $C(z,$.$) is a dilation$ for every $z \in[0,1]$ (the optimistic $t$-norm $\mathcal{T}_{M}^{o}$ associated with the minimum $t$-norm represents an exception because $\mathcal{T}_{M}^{o}=\mathcal{T}_{M}^{r}$ ). As an example consider the optimistic $t$-norm $\mathcal{T}_{P}^{o}$ corresponding to the product $t$-norm on $[0,1] \times[0,1]$.

Let $\mathbf{z}=[0.5,1], \mathbf{x}^{1}=[0,1]$, and $\mathbf{x}^{2}=[0.5,0.7]$. Let us show that $\mathcal{T}_{P}^{o}\left(\mathbf{z}, \mathbf{x}^{1} \vee \mathbf{x}^{2}\right) \neq \mathcal{T}_{P}^{o}\left(\mathbf{z}, \mathbf{x}^{1}\right) \vee \mathcal{T}_{P}^{o}\left(\mathbf{z}, \mathbf{x}^{2}\right)$. If 
$\mathbf{x}$ denotes $\mathbf{x}^{1} \vee \mathbf{x}^{2}=[0.5,1]$ then it suffices to show the following inequality

$z_{1} x_{2} \wedge z_{2} x_{1} \neq\left(z_{1} x_{2}^{1} \wedge z_{2} x_{1}^{1}\right) \vee\left(z_{1} x_{2}^{2} \wedge z_{2} x_{1}^{2}\right)$.

The left-hand side of this expression equals 0.5 whereas the right-hand side equals $0 \vee 0.35=0.35$. In a similar vein, the pessimistic implication $\mathcal{I}_{I}^{p}$ does generally not yield an erosion for fixed $\mathbf{z} \in \mathbb{L}^{I}$ even if $I(z,$.$) is an erosion for$ every $z \in[0,1]$.

The next two sections are concerned with the types of interval-valued fuzzy connectives that arise in applications of the duality relationships of negations and adjunction or, more generally, in applications of Equations 10 and 12 - to representable and pseudo-representable interval-valued fuzzy connectives.

\subsubsection{Construction of Operators Using Adjunction}

Under certain conditions stated in Theorem 1, we can derive the $R$-implication of a given interval-valued fuzzy conjunction $\mathcal{C}$ and the $R$-conjunction of a given interval-valued fuzzy implication $\mathcal{I}$. As mentioned above, this construction leads to an adjunction $(\mathcal{I}, \mathcal{C})$ if we are given a conjunction $\mathcal{C}$ on $\mathbb{L}^{I}$ that constitutes a dilation in the second argument or an implication $\mathcal{I}$ on $\mathbb{L}^{I}$ that constitutes an erosion in the second argument. Some of the special cases discussed in the previous section are especially easy to deal with:

Theorem 13 If $C$ is a fuzzy conjunction such that $C(1, x)>$ 0 for all $x \neq 0$ then the $R$-implication of the pessimistic conjunction $\mathcal{C}_{C}^{p}$ is given by the optimistic implication $\mathcal{I}_{I}^{o}$ where $I$ is the $R$-implication of $C$. In this case, we also have that $\mathcal{I}_{I}^{o}$ and $\mathcal{C}_{C}^{p}$ are adjoint if and only if $I$ and $C$ are adjoint.

Conversely, if I is a fuzzy implication such that $I(1, x)<$ 1 for all $x \neq 1$ then the $R$-conjunction of the optimistic implication $\mathcal{I}_{I}^{o}$ is given by the pessimistic conjunction $\mathcal{C}_{C}^{p}$ where $C$ is the $R$-conjunction of $I$. In this case, we also have that $\mathcal{I}_{I}^{o}$ and $\mathcal{C}_{C}^{p}$ are adjoint if and only if $I$ and $C$ are adjoint.

For example, the adjoint implication of $\mathcal{T}_{L}=\mathcal{T}_{W}^{p}$ is given by $\mathcal{I}_{W}^{o}$, i.e., the optimistic implication with the representative $I_{W}$ and the adjoint implication of $\mathcal{C}_{K}^{p}$ is given by $\mathcal{I}_{K}^{o}$, i.e., the optimistic implication with the representative $I_{K}$, where $I_{K}$ denotes the Kleene implicator.

Theorem 14 If $C$ and $C^{\prime}$ are fuzzy conjunctions such that $C(x, y) \leq C^{\prime}(x, y)$ for all $x, y \in[0,1]$ and $C^{\prime}(1, x)>0$ for all $x \neq 0$ then the $R$-implication of the representable fuzzy conjunction $\mathcal{C}_{C, C^{\prime}}^{r}$ is given by the following expression where $I$ is the R-implication of $C$ and $I^{\prime}$ is the $R$ implication of $C^{\prime}$ :

$\mathcal{I}_{\mathcal{C}_{C, C^{\prime}}^{r}}(\mathbf{x}, \mathbf{y})=\left[I\left(x_{1}, y_{1}\right) \wedge I^{\prime}\left(x_{2}, y_{2}\right), I^{\prime}\left(x_{2}, y_{2}\right)\right]$.
In this case, $\mathcal{C}_{C, C^{\prime}}^{r}$ and its $R$-implication are adjoint if and only if the pairs $(I, C)$ and $\left(I^{\prime}, C^{\prime}\right)$ form adjunctions. Similarly, if $I$ and $I^{\prime}$ are fuzzy implications such that $I(x, y)$ $\leq I^{\prime}(x, y)$ for all $x, y \in[0,1]$ and $I(1, x)<1$ for all $x \neq 1$ then the $R$-conjunction of the representable fuzzy implication $\mathcal{I}_{I, I^{\prime}}^{r}$ is given by the following expression where $C$ is the $R$-conjunction of $I$ and $C^{\prime}$ is the $R$-conjunction of $I^{\prime}$.

$\mathcal{C}_{\mathcal{I}_{I, I^{\prime}}^{r}}(\mathbf{x}, \mathbf{y})=\left[C\left(x_{2}, y_{1}\right), C\left(x_{2}, y_{1}\right) \vee C^{\prime}\left(x_{1}, y_{2}\right)\right]$

In this case, $\mathcal{I}_{I, I^{\prime}}^{r}$ and its $R$-conjunction are adjoint if and only if the pairs $(I, C)$ and $\left(I^{\prime}, C^{\prime}\right)$ form adjunctions.

Let us apply Theorem 14 to the interval-valued minimum operator $\mathcal{T}_{M}=\mathcal{T}_{M}^{r}$. Recall that the fuzzy minimum $T_{M}$ is adjoint to the Gödel implicator $I_{M}$. Therefore, Theorem 12 reveals that the $R$-implication of $\mathcal{T}_{M}$ forms an adjunction with $\mathcal{T}_{M}$. By Theorem 14, we obtain $\mathcal{I}_{\mathcal{T}_{M}}(\mathbf{x}, \mathbf{y})=$ $\left[I_{M}\left(x_{1}, y_{1}\right) \wedge I_{M}\left(x_{2}, y_{2}\right), I_{M}\left(x_{2}, y_{2}\right)\right]$. Closer inspection shows that the latter expression boils down to the intervalvalued implication $\mathcal{I}_{S}$ that was introduced in Equation 43.

The $R$-implications of optimistic conjunctions and the $R$-conjunctions of pessimistic implications are also neither representable nor pseudo-representable. Let us for instance derive the $R$-implication of the optimistic conjunction $C_{C}^{o}$ with representative $C$. The condition $C(1, x)>0$ for all $x \neq 0$ guarantees the existence of this $R$-implication that is given as follows in terms of the $R$-implication $I$ of the fuzzy conjunction $C$ :

$$
\begin{aligned}
& \mathcal{I}_{\mathcal{C}_{C}^{o}}(\mathbf{z}, \mathbf{y})=\bigvee\left\{\mathbf{x} \in \mathbb{L}^{I}: \mathcal{C}_{C}^{o}(\mathbf{z}, \mathbf{x}) \leq \mathbf{y}\right\}= \\
& \bigvee\left\{\mathbf{x} \in \mathbb{L}^{I}:\left[C\left(z_{1}, x_{2}\right) \wedge C\left(z_{2}, x_{1}\right), C\left(z_{2}, x_{2}\right)\right] \leq\left[y_{1}, y_{2}\right]\right\} \\
& =\bigvee\left\{\mathbf{x} \in \mathbb{L}^{I}:\left(C\left(z_{1}, x_{2}\right) \leq y_{1} \text { or } C\left(z_{2}, x_{1}\right) \leq y_{1}\right)\right. \\
& \text { and } \left.C\left(z_{2}, x_{2}\right) \leq y_{2}\right\} \\
& =\bigvee\left\{\mathbf{x} \in \mathbb{L}^{I}:\left(C\left(z_{1}, x_{2}\right) \leq y_{1} \text { and } C\left(z_{2}, x_{2}\right) \leq y_{2}\right)\right. \\
& \text { or } \left.\left(C\left(z_{2}, x_{1}\right) \leq y_{1} \text { and } C\left(z_{2}, x_{2}\right) \leq y_{2}\right)\right\} \\
& =\bigvee\left\{\mathbf{x} \in \mathbb{L}^{I}:\left(C\left(z_{1}, x_{1}\right) \leq y_{1} \text { and } C\left(z_{2}, x_{1}\right) \leq y_{2}\right.\right. \\
& \text { and } \left.C\left(z_{1}, x_{2}\right) \leq y_{1} \text { and } C\left(z_{2}, x_{2}\right) \leq y_{2}\right) \\
& \text { or } \left.\left(C\left(z_{2}, x_{1}\right) \leq y_{1} \text { and } C\left(z_{2}, x_{2}\right) \leq y_{2}\right)\right\} \\
& =\bigvee\left\{\mathbf{x} \in \mathbb{L}^{I}: C\left(z_{1}, x_{1}\right) \leq y_{1} \text { and } C\left(z_{2}, x_{1}\right) \leq y_{2}\right. \\
& \text { and } \left.C\left(z_{1}, x_{2}\right) \leq y_{1} \text { and } C\left(z_{2}, x_{2}\right) \leq y_{2}\right\} \\
& \vee \bigvee\left\{\mathbf{x} \in \mathbb{L}^{I}: C\left(z_{2}, x_{1}\right) \leq y_{1} \text { and } C\left(z_{2}, x_{2}\right) \leq y_{2}\right\} \\
& =\left[\bigvee\left\{x_{1} \in[0,1]: C\left(z_{1}, x_{1}\right) \leq y_{1} \text { and } C\left(z_{2}, x_{1}\right) \leq y_{2}\right\}\right. \text {, } \\
& \left.\bigvee\left\{x_{2} \in[0,1]: C\left(z_{1}, x_{2}\right) \leq y_{1} \text { and } C\left(z_{2}, x_{2}\right) \leq y_{2}\right\}\right] \\
& \vee\left[\bigvee\left\{x_{1} \in[0,1]: C\left(z_{2}, x_{1}\right) \leq y_{1}\right\},\right. \\
& \left.\bigvee\left\{x_{2} \in[0,1]: C\left(z_{2}, x_{2}\right) \leq y_{2}\right\}\right]
\end{aligned}
$$




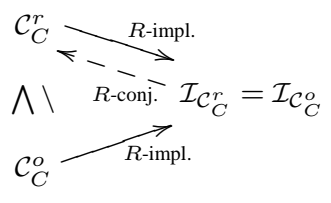

$$
\begin{aligned}
& \mathcal{I}_{I}^{r} \underset{-R \text {-conj. }}{\longrightarrow}
\end{aligned}
$$

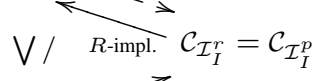

$$
\begin{aligned}
& \mathcal{I}_{I}^{p}--\underset{R \text {-conj. }}{\stackrel{7}{ }}
\end{aligned}
$$

Fig. 1 Relationships between some representable and pseudorepresentable logical connectives on $\mathbb{L}^{I}$ and their $R$-implications and $R$-conjunctions.

$$
\begin{aligned}
= & {\left[I\left(z_{1}, y_{1}\right) \wedge I\left(z_{2}, y_{2}\right), I\left(z_{1}, y_{1}\right) \wedge I\left(z_{2}, y_{2}\right)\right] } \\
& \vee\left[I\left(z_{2}, y_{1}\right), I\left(z_{2}, y_{2}\right)\right] \\
= & {\left[\left(I\left(z_{2}, y_{1}\right) \vee I\left(z_{1}, y_{1}\right)\right)\right.} \\
& \left.\wedge\left(I\left(z_{2}, y_{1}\right) \vee I\left(z_{2}, y_{2}\right)\right), I\left(z_{2}, y_{2}\right)\right] \\
= & {\left[I\left(z_{1}, y_{1}\right) \wedge I\left(z_{2}, y_{2}\right), I\left(z_{2}, y_{2}\right)\right] }
\end{aligned}
$$

Similarly, if $I(1, x)<1$ for all $x \neq 1$ then $\mathcal{C}_{\mathcal{I}_{I}^{p}}$, the $R$ conjunction of the pessimistic implication $\mathcal{I}_{I}^{p}$ is given by the following expression where $C$ is the $R$-conjunction of $I$ :

$\mathcal{C}_{\mathcal{I}_{I}^{p}}(\mathbf{z}, \mathbf{x})=\left[C\left(z_{2}, x_{1}\right), C\left(z_{2}, x_{1}\right) \vee C\left(z_{1}, x_{2}\right)\right] \forall \mathbf{z}, \mathbf{x} \in \mathbb{L}^{I}$

Note that $\mathcal{C}_{C}^{r}$ and $\mathcal{C}_{C}^{o}$ possess the same $R$-implications and that $\mathcal{I}_{I}^{r}$ and $\mathcal{I}_{I}^{p}$ possess the same $R$-conjunctions. These facts do not contradict the uniqueness property of the adjoint operator since - as mentioned before $-\mathcal{C}_{C}^{o}(\mathbf{z},$.$) gen-$ erally does not constitute a dilation and since $\mathcal{I}_{I}^{p}(\mathbf{z},$.$) does$ generally not constitute an erosion. Even if $C(z,$.$) are dila-$ tions and $I(z,$.$) are erosions for all z \in[0,1]$ we have that generally neither $\mathcal{C}_{C}^{o}$ and its $R$-implication nor $\mathcal{I}_{I}^{P}$ and its $R$-conjunction form an adjunction.

However, a fuzzy conjunction $C$ with dilative partial mappings $C(z,$.$) induces the C$-representable conjunction $\mathcal{C}_{C}^{r}$ whose partial mappings $\mathcal{C}_{C}^{r}(\mathbf{z},$.$) are also dilations according$ to Theorem 12. Thus, $\mathcal{C}_{C}^{r}$ and its $R$-implication $\mathcal{I}_{\mathcal{C}_{C}^{r}}=\mathcal{I}_{\mathcal{C}_{C}^{o}}$ form an adjunction and the $R$-conjunction of $\mathcal{I}_{\mathcal{C}_{C}^{r}}$ is given by $\mathcal{C}_{C}^{r}$. In addition, Theorem 3 implies $\mathcal{C}_{C}^{r} \leq \mathcal{C}_{C}^{o}$. Similar comments can be made with respect to $\mathcal{I}_{I}^{r}, \mathcal{I}_{I}^{p}$, and their common $R$-conjunction provided that $I$ has erosive partial mappings $I(z,$.$) for all z \in[0,1]$. Figure 1 illustrates these observations.

Of course, the construction schemes based on Equations 10 and 12 can also be applied to logical connectives on $\mathbb{L}^{I}$ or $\mathbb{L}^{*}$ that are neither representable nor pseudo-representable. For example, forming the $R$-conjunction of the implication $\mathcal{I}_{M}$ given in Equation 41 yields the following $R$-conjunction $\mathcal{C}_{M}$ :

$$
\mathcal{C}_{M}(\mathbf{x}, \mathbf{y})= \begin{cases}\mathbf{x} & \text { if } \mathbf{x} \leq \mathbf{y} \\ \mathbf{y} & \text { else }\end{cases}
$$

Note that $\mathcal{C}_{M}$ is not a $t$-norm on $\mathbb{L}^{I}$. Moreover, the unary operators $\mathcal{C}_{M}(\mathbf{z},$.$) do not represent dilations for all \mathbf{z} \in \mathbb{L}^{I}$ which can be seen as follows. For $\mathbf{z}=[0.3,0.8], \mathbf{x}^{1}=$ $[0.2,1]$, and $\mathbf{x}^{2}=[0.4,1]$, let $\mathbf{x}=\mathbf{x}^{1} \vee \mathbf{x}^{2}=[0.4,1]$. We have $\mathcal{C}_{M}(\mathbf{z}, \mathbf{x})=\mathbf{z}=[0.3,0.8] \neq[0.4,1]=\mathcal{C}_{M}\left(\mathbf{z}, \mathbf{x}^{1}\right) \vee$ $\mathcal{C}_{M}\left(\mathbf{z}, \mathbf{x}^{2}\right)$. Therefore $\mathcal{I}_{M}$ and $\mathcal{C}_{M}$ are not adjoint.

The next section is concerned with the construction of interval-valued operators from given interval-valued operators using the duality relationship of negation.

\subsubsection{Construction of Operators Using Negation}

By Theorem 4, we can employ interval-valued negations in order to build new interval-valued implications, conjunctions, and disjunctions from given interval-valued fuzzy operators. Although this section focusses on interval-valued implications and conjunctions (the underlying operators of interval-valued fuzzy MM) the subsequent results can be easily extended to include interval-valued disjunctions. Due to the isomorphism between $\mathbb{L}^{I}$ and $\mathbb{L}^{*}$, applications of Theorems 9 and 10 yield analogous results for the intuitionistic fuzzy case.

According to Deschrijver, Cornelis, and Kerre [25,26], every negation $\mathcal{N}$ on $\mathbb{L}^{I}$ is determined by a fuzzy negation $N$, called representative of $\mathcal{N}$. Specifically, every negation on $\mathbb{L}^{I}$ can be written in the form $\mathcal{N}_{N}$ where $\mathcal{N}_{N}(\mathbf{x})=$ $\left[N\left(x_{2}\right), N\left(x_{1}\right)\right]$ for every $\mathbf{x} \in \mathbb{L}^{I}$.

First, let us take a look at the $\mathcal{N}_{N}$-dual operators of representable and pseudo-representable operators on $\mathbb{L}^{I} \times \mathbb{L}^{I}$. This issue has already been addressed by Deschrijver and Cornelis who have formed $S$-implicators of optimistic, pessimistic, and $t$-representable $s$-norms with a single representative [25].

Theorem 15 Let $N$ be a fuzzy negation. If $C$ and $C^{\prime}$ are fuzzy conjunctions such that $C(x, y) \leq C^{\prime}(x, y)$ for all $x, y \in$ $[0,1]$ and if $I$ and $I^{\prime}$ are fuzzy implications such that $I^{\prime}(x, y) \leq$ $I(x, y)$ for all $x, y \in[0,1]$ then the following statement holds true:

The $C$-representable conjunction $\mathcal{C}_{C, C^{\prime}}^{r}$ and the I-representable implication $\mathcal{I}_{I^{\prime}, I}^{r}$ are dual with respect to the intervalvalued fuzzy negation $\mathcal{N}_{N}$ if and only if $C$ and I as well as $C^{\prime}$ and $I^{\prime}$ are dual with respect to $N$.

From now on, let $I_{R}$ and $I_{M}$ denote respectively the Reichenbach and the Gödel implicators. By Theorem 15, the pairs $\left(\mathcal{T}_{M}^{r}, \mathcal{I}_{K}^{r}\right),\left(\mathcal{T}_{P}^{r}, \mathcal{I}_{R}^{r}\right),\left(\mathcal{T}_{W}^{r}, \mathcal{I}_{W}^{r}\right)$, and $\left(\mathcal{C}_{K}^{r}, \mathcal{I}_{M}^{r}\right)$ exemplify pairwise dual operators with respect to the standard interval-valued negation $\mathcal{N}_{S}$ having a single representative. The pairs $\left(\mathcal{T}_{P, M}^{r}, \mathcal{I}_{K, R}^{r}\right)$ and $\left(\mathcal{C}_{K, M}^{r}, \mathcal{I}_{K, G}^{r}\right)$ provide examples of pair-wise dual operators with respect to $\mathcal{N}_{S}$ having two distinct representatives.

Theorem 16 Let $C$ be a fuzzy conjunction, let I be a fuzzy implication, and let $N$ be a fuzzy negation. The pessimistic 
conjunction $\mathcal{C}_{C}^{p}$ and the optimistic implication $I_{I}^{o}$ are dual with respect to $\mathcal{N}_{N}$ if and only if $C$ and I are dual with respect to $N$. The optimistic conjunction $\mathcal{C}_{C}^{o}$ and the pessimistic implication $I_{I}^{p}$ are dual with respect to $\mathcal{N}_{N}$ if and only if $C$ and I are dual with respect to $N$.

Theorem 16 leads to the following pairs of dual operators with respect to $\mathcal{N}_{S}$ :

- $\left(\mathcal{T}_{M}^{p}, \mathcal{I}_{K}^{o}\right),\left(\mathcal{T}_{P}^{p}, \mathcal{I}_{R}^{o}\right),\left(\mathcal{T}_{W}^{p}, \mathcal{I}_{W}^{o}\right)$, and $\left(\mathcal{C}_{K}^{p}, \mathcal{I}_{M}^{o}\right)$;

- $\left(\mathcal{T}_{M}^{o}, \mathcal{I}_{K}^{p}\right),\left(\mathcal{T}_{P}^{o}, \mathcal{I}_{R}^{p}\right),\left(\mathcal{T}_{W}^{o}, \mathcal{I}_{W}^{p}\right)$, and $\left(\mathcal{C}_{K}^{o}, \mathcal{I}_{M}^{p}\right)$.

This way we can construct a host of pairs that consist of dual interval-valued operators with respect to a certain interval-valued negation. Of course, this construction scheme based on negation is not limited to representable or pseudorepresentable fuzzy connectives but can be applied to any interval-valued fuzzy implication, conjunction, or disjunction.

For example, forming the $\mathcal{N}_{S}$-dual of the interval-valued implication $\mathcal{I}_{M}$ introduced in Equation 41 yields the following interval-valued conjunction:

$\mathcal{C}_{\mathcal{I}_{M}, \mathcal{N}_{S}}(\mathbf{x}, \mathbf{y})= \begin{cases}0_{\mathbb{L}^{I}} & \text { if } \mathbf{x} \leq \mathcal{N}_{S}(\mathbf{y}) \\ \mathbf{y} & \text { else }\end{cases}$

In conclusion, the four strategies presented in Section 4.2 lead to a large variety of interval-valued and intuitionistic fuzzy implications and conjunctions that can serve as the building blocks for particular approaches to interval-valued or intuitionistic FMM. The choice of an appropriate pair of particular interval-valued or intuitionistic fuzzy connectives for a given application represents an open research problem, just as in the classical fuzzy case.

In some previous papers [57,59], we have provided some visual examples of applications of interval-valued dilations, erosions, and edge detection (dilation minus erosion). We chose to use the interval-valued fuzzy dilation $\Delta_{W}^{p}$ that is based on $\mathcal{T}_{W}^{p}$ and the interval-valued fuzzy erosion $\mathcal{E}_{W}^{o}$ that is based on $\mathcal{I}_{W}^{o}$ because $\Delta_{W}^{p}$ and $\mathcal{E}_{W}^{o}$ are both adjoint and dual with respect to $\mathcal{N}_{S}$ by Theorem 7. For the same reasons, these interval-valued fuzzy operators were also selected for the following simulations concerning tomographic image reconstruction methods.

\section{Some Experimental Results Concerning Tomographic Image Reconstruction}

The morphological watershed transform can be classified as a region-based approach for image segmentation [63]. The literature on morphological image processing abounds with versions of the watershed transforms. In practice, the watershed transform is often applied to the (morphological) gradient of the original image [55]. In addition, some pre- or postprocessing techniques are ususally employed to avoid oversegmentation. In all the gradient and filtering techniques we applied in the following experiments we used the 8-connected disk with radius 1 as a structuring element.

Two of the most widely used watershed algorithms are the flooding algorithm of F. Meyer based on the concept of topographical distance and the recursive immersion algorithm of Vincent and Soille [54,86]. In the following experiments we applied the MATLAB implementation of F. Meyer's algorithm to the (post-processed versions of) morphological gradients of images that arose by applying three well-known image reconstruction techniques to the so called Shepp-Logan phantom. In addition, we generated a single interval-valued image by combining the three reconstructed images and computed the mean of the upper and the lower envelope of a certain interval-valued morphological gradient. After some post-processing, the resulting image was segmented using Meyer's algorithm.

Let us now describe the details of our experiments. Figure 2 displays a discretized version of the famous SheppLogan phantom (on a $256 \times 256$ grid) [43] as well as the reconstructions produced by the following algorithms [40, 82]: filtered backprojection (FBP), filter of the backprojections (FOB), and Tretiak \& Metz reconstruction. These algorithms were executed in a noiseless setting using 600 uniform views and 400 equally spaced rays within each view. The Ramlak filter [43] was employed in both the FBP and the FOB algorithm. The attenuation parameter for the TretiakMetz inversion was set to 0.1 . The morphological gradients of these images were calculated by subtracting the eroded images from the respective dilated images. Subsequently, we filtered these gradient images by applying the $h$-minima transform with $h=0.07$ [74] and computed the watershed segmentation corresponding to each of the individual images. The filtered morphological gradient images and the watershed images are shown respectively in Figures 3 and 4.

We also combined the three image reconstruction methods mentioned above by constructing an interval-valued fuzzy image as follows. The lower and upper bounds shown in Figure 5 are given by respectively the pixelwise minimum and maximum of the three reconstructed images. Then we computed the interval-valued morphological gradient in terms of interval-valued fuzzy dilation $\Delta_{W}^{p}$ based on $\mathcal{T}_{W}^{p}$ and the interval-valued fuzzy erosion $\mathcal{E}_{W}^{o}$ based on $\mathcal{I}_{W}^{o}$. The difference operator that occurs in the interval-valued morphological gradient was chosen as follows [25]:

$\mathbf{x}-\mathbf{y}=\left[x_{1}-y_{2},\left(x_{1}-y_{1}\right) \vee\left(x_{2}-y_{2}\right)\right] \forall \mathbf{x}, \mathbf{y} \in \mathbb{L}^{I}$ 

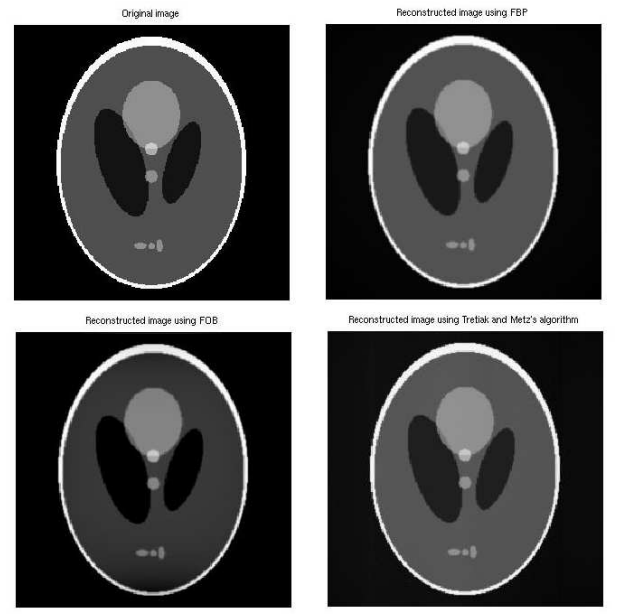

Fig. 2 Original Shepp-Logan phantom and reconstructions produced by the FBP, FOB, and Tretiak \& Metz algorithms.
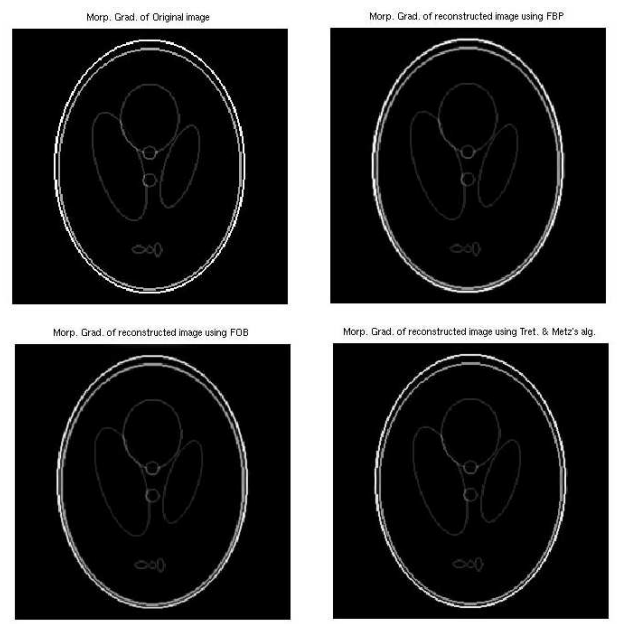

Fig. 3 Morphological gradients of the Shepp-Logan phantom and of the reconstructions produced by the FBP, FOB, and Tretiak \& Metz algorithms after applications of the $h$-minima transform.

We then formed the mean of the lower and upper bounds of the interval-valued gradient image depicted in the top row of Figure 6 and subjected it to the $h$-minima transform using the same parameter of $h=0.07$ as above. Finally, F. Meyer's algorithm was applied to the resulting image which is shown as the first image of the bottom row of Figure 6. The image on the right side of the second row of Figure 6 illustrates the watershed segmentation corresponding to the combination of the three image reconstruction methods. Close visual inspection reveals that this result which is based on the interval-valued combimation of the three methods is slightly more similar to the segmentation of the original image shown in Figure 4 than the segmentation results obtained by the individual methods.
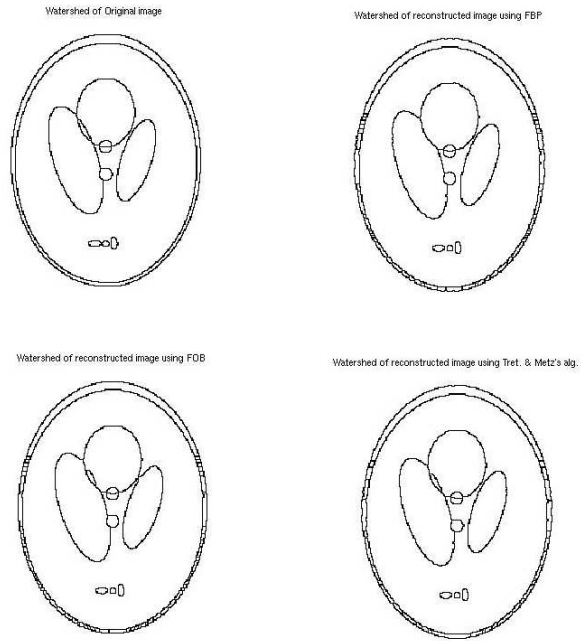

Fig. 4 Watershed transform of Shepp-Logan phantom and of reconstructions produced by the FBP, FOB, and Tretiak \& Metz algorithms.
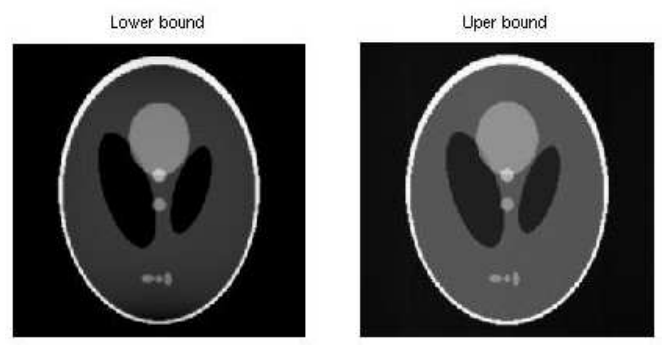

Fig. 5 Lower and upper bounds of the interval-valued representation given by the pixelwise minimum and maximum of the three reconstructed images in Figure 2.

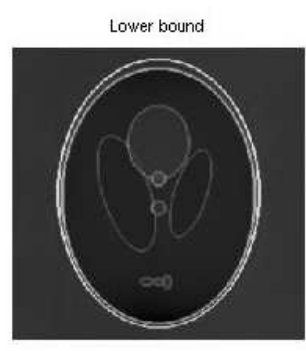

Mean of Morp. Grad.

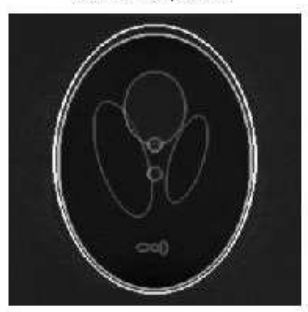

Upper bound

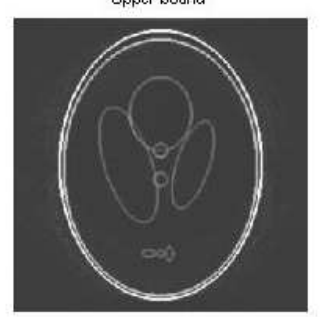

Watershed of mean of Morp. Grad.

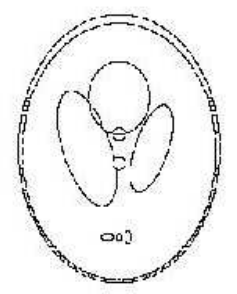

Fig. 6 The top row shows the lower and upper bounds of the intervalvalued morphological gradient of the image in Figure 5 based on $\Delta_{W}^{p}$ and $\mathcal{E}_{W}^{o}$. The image on the left of the bottom row depicts the mean of the images on top after transforming it using $h$-minima. The last image depicts the result after applying the watershed transform. 


\section{Concluding Remarks}

This paper introduces $\mathbb{L}$-fuzzy $\mathrm{MM}$ where $\mathbb{L}$ is an arbitrary complete lattice. We have described general aspects of $\mathbb{L}$ fuzzy MM such as the construction of $\mathbb{L}$-fuzzy erosion and dilation based on logical connectives on $\mathbb{L}$ and relationships of duality in $\mathbb{L}$-fuzzy MM. Interval-valued and intuitionistic FMMs arise as special cases of $\mathbb{L}$-fuzzy MM. In this context, we presented several strategies for constructing interval-valued and intuitionistic fuzzy logical connectives. We believe that the results of this paper can be the starting point for fruitful research endeavors in the following areas:

1. Applications of interval-valued and intuitionistic FMM in image processing: As we have pointed out before [5759], interval-valued and intuitionistic fuzzy set theory enable us to model numerical and spatial uncertainty in grayscale images that is due to image capture, leading to specific morphological operators and related applications that have yet to be explored in detail. In previous papers, we provided some preliminary results concerning interval-valued edge detection [57-59]. In this paper, we went one step further by outlining an application of interval-valued FMM that aims at combining different methods for medical image reconstruction in terms of the watershed transform. Clearly, the approach presented in this paper only represents our first attempt to tackle this problem using the emerging techniques of intervalvalued FMM. Nevertheless, we believe that every existing application of gray-scale or fuzzy MM in image processing potentially lends itself to interval-valued FMM if some uncertainty regarding the pixel values exists. In contrast to conventional morphological techniques, interval-valued FMM techniques are able to keep track of this uncertainty information.

Another interpretation of bipolar FMM (i.e., intuitionistic FMM) was provided by I. Bloch who has presented applications to spatial reasoning in image processing [11, 13]. In this context, one distinguishes between positive (representing what is granted to be possible) and negative (representing what is impossible) information, whose intersection has to be empty and whose union does not necessarily cover the whole underlying space.

2. Development of $\mathbb{L}$-fuzzy MMs for other special cases of complete lattices $\mathbb{L}$ : Conventional, interval-valued, and intuitionistic fuzzy sets represent particular instances of information granules $[8,90]$. We suspect that there are other specific classes of information granules that form complete lattices and that are conducive to the application of morphological tools [81].

3. Development of $\mathbb{L}-f u z z y$ - in particular interval-valued and intuitionistic fuzzy - extensions of existing morphological neural networks such as fuzzy morphological associative memories [76-79, 81,85]: Training of these new models may be achieved by means of a generalization of the "fuzzy learning by adjunction" scheme [85]. This issue appears to be promising due to the large number of applications of interval-valued type- 2 fuzzy sets in rulebased systems and approximate reasoning [26, 83, 84].

\section{A Proofs of Theorems}

\section{Proof of Theorem 1}

1. Let us assume that the conditions of the theorem are satisfied. First, let us prove Part 1.

(a) $I_{C}(., y)$ is decreasing for all $y \in \mathbb{L}$ :

Consider an arbitrary element $y$ of $\mathbb{L}$. Let us compare the sets $\{x \in \mathbb{L}: C(w, x) \leq y\}$ and $\{x \in \mathbb{L}: C(z, x) \leq y\}$ for arbitrary $w, z \in \mathbb{L}$ such that $w \leq z$. Since $C(w, x) \leq$ $C(z, x)$, we have $\{x \in \mathbb{L}: C(w, x) \leq y\} \supseteq\{x \in \mathbb{L}$ : $C(z, x) \leq y\}$. Hence, we are able to conclude that

$$
I_{C}(w, y)=\bigvee\{x \in \mathbb{L}: C(w, x) \leq y\}
$$$$
\geq \bigvee\{x \in \mathbb{L}: C(z, x) \leq y\}=I_{C}(z, y) \text {. }
$$

(b) $I_{C}(z,$.$) is increasing for all z \in \mathbb{L}$ :

Let $z \in \mathbb{L}$ be arbitrary. Consider arbitrary elements $y$ and $w$ of $\mathbb{L}$ such that $y \leq w$. Since the set $\{x \in \mathbb{L}: C(z, x) \leq y\}$ is contained in the set $\{x \in \mathbb{L}: C(z, x) \leq w\}$, we conclude that

$$
\begin{array}{r}
I_{C}(z, y)=\bigvee\{x \in \mathbb{L}: C(z, x) \leq y\} \\
\leq \bigvee\{x \in \mathbb{L}: C(z, x) \leq w\}=I_{C}(z, w) .
\end{array}
$$

(c) Equation 11 holds:

One one hand, since $C\left(0_{\mathbb{L}}, 1_{\mathbb{L}}\right)=0_{\mathbb{L}}$ and $1_{\mathbb{L}}=\bigvee \mathbb{L}$, we have $I_{C}\left(0_{\mathbb{L}}, y\right)=\bigvee\left\{x \in \mathbb{L}: C\left(0_{\mathbb{L}}, x\right) \leq y\right\}=1_{\mathbb{L}}$ for all $y \in \mathbb{L}$, in particular for $y=0_{\mathbb{L}}$ and $y=1_{\mathbb{L}}$. On the other hand, since $C\left(1_{\mathbb{L}}, 1_{\mathbb{L}}\right)=1_{\mathbb{L}}$ and $1_{\mathbb{L}}=\bigvee \mathbb{L}$, we have $I_{C}\left(1_{\mathbb{L}}, 1_{\mathbb{L}}\right)=\bigvee\left\{x \in \mathbb{L}: C\left(1_{\mathbb{L}}, x\right) \leq 1_{\mathbb{L}}\right\}=1_{\mathbb{L}}$.

2. For the proof of Part 2, note that $I_{C}$ as defined in Equation 10 satisfies all the requirements of an $\mathbb{L}$-fuzzy implication except $I_{C}\left(1_{\mathbb{L}}, 0_{\mathbb{L}}\right)$ $=0_{\mathbb{L}}$. Thus, the function $I_{C}$ represents an $\mathbb{L}$-fuzzy implication if and only if $0_{\mathbb{L}}=I_{C}\left(1_{\mathbb{L}}, 0_{\mathbb{L}}\right)=\bigvee\left\{x \in \mathbb{L}: C\left(1_{\mathbb{L}}, x\right) \leq 0_{\mathbb{L}}\right\}$. Equivalently, we have $C\left(1_{\mathbb{L}}, x\right)>0_{\mathbb{L}}$ for all $x \in \mathbb{L} \backslash\left\{0_{\mathbb{L}}\right\}$ since $0_{\mathbb{L}}=\bigwedge \mathbb{L}$.

\section{Proof of Theorem 2}

The proof is similar to the one of Theorem 1.

\section{Proof of Theorem 3}

Let $C$ and $I_{C}$ be as stated above. Note that the existence of the $R$ implication of $C$ and of the $R$-conjunction of $I_{C}$ follows from Theorems 1 and 2. If $C^{\prime}$ denotes the $R$-conjunction of $I_{C}$ then $C^{\prime}$ can be computed as follows for all $z, x \in \mathbb{L}$ :

$$
\begin{array}{r}
C^{\prime}(z, x)=\bigwedge\left\{y \in \mathbb{L}: I_{C}(z, y) \geq x\right\} \\
=\bigwedge\left\{y \in \mathbb{L}: \bigvee\left\{x^{\prime} \in \mathbb{L}: C\left(z, x^{\prime}\right) \leq y\right\} \geq x\right\}
\end{array}
$$

Replacing $y$ by $C(z, x)$ in $\bigvee\left\{x^{\prime} \in \mathbb{L}: C\left(z, x^{\prime}\right) \leq y\right\}$, we realize that this supremum is greater than or equal to $x$ because $C$ is increasing. Therefore, $C(z, x)$ is contained in the set over which the infimum is taken in Equation 78 which implies that $C^{\prime}(z, x) \leq C(z, x)$ for all $z, x \in \mathbb{L}$. We omit the second, similar part of the theorem. 


\section{Proof of Theorem 4}

Assume that $I$ is an $\mathbb{L}$-fuzzy implication. Let $C$ be given by Equation 7. Note that the action of $I$ on the set $\left\{0_{\mathbb{L}}, 1_{\mathbb{L}}\right\}$ determines the action of $C$ on the same set. The operator $C$ is increasing in both arguments because $I$ is decreasing in the first argument and increasing in the second argument and because $N$ inverts the partial order. The rest of the theorem can be demonstrated in a similar way.

\section{Proof of Theorem 5}

The equivalence of the first two statements follows from the fact that $\mathcal{E}_{\mathbb{L}}$ is an erosion in the first argument if and only if the following equations are satisfied for all index sets $K, \mathbf{a}_{k}, \mathbf{s} \in \mathcal{F}_{\mathbb{L}}(\mathbf{X})$, and $\mathbf{x} \in \mathbf{X}$ :

$\mathcal{E}_{\mathbb{L}}\left(\bigwedge_{k \in K} \mathbf{a}_{k}, \mathbf{s}\right)(\mathbf{x})=\bigwedge_{k \in K} \mathcal{E}_{\mathbb{L}}\left(\mathbf{a}_{k}, \mathbf{s}\right)(\mathbf{x})$.

Equivalently, we have $\operatorname{Inc}_{\mathbb{L}}\left(\mathbf{s}_{\mathbf{x}}, \bigwedge_{k \in K} \mathbf{a}_{k}\right)=\bigwedge_{k \in K} \operatorname{In} c_{\mathbb{L}}\left(\mathbf{s}_{\mathbf{x}}, \mathbf{a}_{k}\right)$ for all index sets $K$, for all $\mathbf{a}_{k}, \mathbf{s} \in \mathcal{F}_{\mathbb{L}}(\mathbf{X})$, and for all $\mathbf{x} \in X$, i.e., the inclusion measure $I n c_{\mathbb{L}}$ represents an erosion in the second argument.

Now, assume that $\operatorname{Inc}_{\mathbb{L}}(\mathbf{s},$.$) are erosions for all \mathbf{s} \in \mathcal{F}_{\mathbb{L}}(\mathbf{X})$. Let us show that $I(s,$.$) are erosions for all s \in \mathbb{L}$. In other words, we want to show that $I\left(s, \bigwedge_{k \in K} a_{k}\right)=\bigwedge_{k \in K} I\left(s, a_{k}\right)$ for all $I$ and for all $s, a_{k} \in \mathbb{L}$. Consider arbitrary elements $s$ and $a_{k}$ of $\mathbb{L}$ where $k \in K$ for some index set $K$. Let $\mathbf{s}$ and $\mathbf{a}_{k}$ be respectively the constant $\mathbb{L}$-fuzzy SE and the constant $\mathbb{L}$-fuzzy images such that $\mathbf{s}(x)=s$ and $\mathbf{a}_{k}(x)=a_{k}$ for all $x \in \mathbf{X}$ and $k \in K$. The following sequence of equalities reveals that $I$ is an erosion in the second argument:

$$
\begin{gathered}
I\left(s, \bigwedge_{k \in K} a_{k}\right)=\bigwedge_{\mathbf{x} \in \mathbf{X}} I\left(\mathbf{s}(\mathbf{x}),\left(\bigwedge_{k \in K} \mathbf{a}_{k}(\mathbf{x})\right)\right) \\
=I n c_{\mathbb{L}}\left(\mathbf{s}, \bigwedge_{k \in K} \mathbf{a}_{k}\right)=\bigwedge_{k \in K} \operatorname{Inc} c_{\mathbb{L}}\left(\mathbf{s}, \mathbf{a}_{k}\right)= \\
\bigwedge_{k \in K}\left(\bigwedge_{\mathbf{x} \in \mathbf{X}} I\left(\mathbf{s}(\mathbf{x}), \mathbf{a}_{k}(\mathbf{x})\right)\right)=\bigwedge_{k \in K} I\left(s, a_{k}\right)
\end{gathered}
$$

Finally, suppose that $I$ is an erosion in the second argument. It suffices to prove that $I n c_{\mathbb{L}}$ represents an erosion in the second argument to finish the proof of the theorem. Note that $I\left(s, \bigwedge_{k \in K} a_{k}\right)=$ $\bigwedge_{k \in K} I\left(s, a_{k}\right)$ for all $K$ and for all $s, a_{k} \in \mathbb{L}^{I}$.

Let $\mathbf{s}, \mathbf{a}_{k} \in \mathcal{F}_{\mathbb{L}}(\mathbf{X})$ be arbitrary. We conclude the proof of the theorem as follows

$$
\begin{aligned}
& I n c_{\mathbb{L}}\left(\mathbf{s}, \bigwedge_{k \in K} \mathbf{a}_{k}\right)=\bigwedge_{\mathbf{x} \in \mathbf{X}} I\left(\mathbf{s}(\mathbf{x}),\left(\bigwedge_{k \in K} \mathbf{a}_{k}\right)(\mathbf{x})\right) \\
& =\bigwedge_{\mathbf{x} \in \mathbf{X}} I\left(\mathbf{s}(\mathbf{x}), \bigwedge_{k \in K} \mathbf{a}_{k}(\mathbf{x})\right)=\bigwedge_{\mathbf{x} \in \mathbf{X}}\left(\bigwedge_{k \in K} I\left(\mathbf{s}(\mathbf{x}), \mathbf{a}_{k}(\mathbf{x})\right)\right) \\
& =\bigwedge_{k \in K}\left(\bigwedge_{\mathbf{x} \in \mathbf{X}} I\left(\mathbf{s}(\mathbf{x}), \mathbf{a}_{k}(\mathbf{x})\right)\right)=\bigwedge_{k \in K} I n c_{\mathbb{L}}\left(\mathbf{s}, \mathbf{a}_{k}\right) .
\end{aligned}
$$

\section{Proof of Theorem 6}

The proof is similar to the one of Theorem 5 .

\section{Proof of Theorem 7}

The first part of Theorem 7 represents a generalization of a proposition that appeared in [24]. We proceed by proving the second part of the theorem. First note that the following statements are equivalent:

- $\mathcal{E}_{\mathbb{L}}$ and $\Delta_{\mathbb{L}}$ are dual with respect to $N$;

- $\mathcal{E}_{\mathbb{L}}(., \mathbf{s})$ and $\Delta_{\mathbb{L}}(., \overline{\mathbf{s}})$ are dual with respect to $N$ for all $\mathbf{s} \in \mathcal{F}_{\mathbb{L}}(\mathbf{X})$;

- $\Delta_{\mathbb{L}}(., \overline{\mathbf{s}})=\mathcal{E}_{\mathbb{L}}(., \mathbf{s})^{\mathcal{N}}$ for all $\mathbf{s} \in \mathcal{F}_{\mathbb{L}}(\mathbf{X})$;

- $\Delta_{\mathbb{L}}(\mathbf{a}, \overline{\mathbf{s}})=\mathcal{N}\left(\mathcal{E}(\mathcal{N}(\mathbf{a}), \mathbf{s})\right.$ for all $\mathbf{s}, \mathbf{a} \in \mathcal{F}_{\mathbb{L}}(\mathbf{X}) ;$
From the definitions of $\mathcal{E}_{\mathbb{L}}$ and $\Delta_{\mathbb{L}}$ and the fact that the bijection $\mathcal{N}$ reverses the partial ordering, we have that $\mathcal{E}_{\mathbb{L}}$ and $\Delta_{\mathbb{L}}$ are dual with respect to $N$ if and only if

$$
\begin{aligned}
& \bigvee_{\mathbf{y} \in \mathbf{X}} C\left(\mathbf{s}_{\mathbf{x}}(\mathbf{y}), \mathbf{a}(\mathbf{y})\right)=N\left(\bigwedge_{\mathbf{y} \in \mathbf{X}} I\left(\mathbf{s}_{\mathbf{x}}(\mathbf{y}), N(\mathbf{a}(\mathbf{y}))\right)\right. \\
& =\bigvee_{\mathbf{y} \in \mathbf{X}}\left(N \left(I\left(\mathbf{s}_{\mathbf{x}}(\mathbf{y}), N(\mathbf{a}(\mathbf{y}))\right) \forall \mathbf{s}, \mathbf{a} \in \mathcal{F}_{\mathbb{L}}(\mathbf{X}), \forall \mathbf{x} \in \mathbf{X} .\right.\right.
\end{aligned}
$$

Equation 82 reveals that if $I$ and $C$ are dual with respect to an $\mathbb{L}$ fuzzy negation $\mathcal{N}$ then $\mathcal{E}_{\mathbb{L}}$ and $\Delta_{\mathbb{L}}$ are dual with respect to $N$. For the proof of the other direction, consider arbitrary $s, a \in \mathbb{L}$. Assume that Equation 82 holds for all $\mathbf{s}, \mathbf{a} \in \mathcal{F}_{\mathbb{L}}(\mathbf{X})$ and for all $\mathbf{x} \in \mathbf{X}$. It suffices to show that $C(s, a)=N(I(s, N(a)))$. Let us choose constant functions $\mathbf{s}$ and $\mathbf{a}$ such that $\mathbf{s}=s$ and such that $\mathbf{a}=a$. Let $\mathbf{x}$ be an arbitrary element of the point set $\mathbf{X}$. We are able to finish the proof of the theorem as follows:

$$
\begin{aligned}
& C(s, a)=\bigvee_{\mathbf{y} \in \mathbf{X}} C(\mathbf{s}(\mathbf{x}-\mathbf{y}), \mathbf{a}(\mathbf{y})) \\
& =\Delta_{\mathbb{L}}(\mathbf{a}, \overline{\mathbf{s}})(\mathbf{x})=\mathcal{N}\left(\mathcal{E}_{\mathbb{L}}(\mathcal{N}(\mathbf{a}), \mathbf{s})\right)(\mathbf{x}) \\
& =N\left(\bigwedge_{\mathbf{y} \in \mathbf{X}} I(\mathbf{s}(\mathbf{y}-\mathbf{x}), N(\mathbf{a}(\mathbf{y})))=N(I(s, N(a))) .\right.
\end{aligned}
$$

\section{Proof of Theorem 8}

Direct verification reveals that $\mathcal{M}^{-1}$ is given by Equation 39. The proofs of Part 1 and 3 follow immediately from the fact that $\phi$ and $\phi^{-1}$ are isomorphisms. The proof of Part 2 additionally uses the commutativity and associativity of $t$-norms and $s$-norms on $\mathbb{L}^{*}$ to infer the commutativity and associativity of the corresponding operators on $\mathbb{L}^{I}$ and vice-versa.

\section{Proof of Theorem 9}

The proof of Theorem 9 resembles the proof of Theorem 8 .

\section{Proof of Theorem 10}

First note that Definition 2 refers to conjunctions, disjunctions, and implications. Therefore, each of the intuitionistic fuzzy connectives $\mathcal{F}^{*}$ and $\mathcal{G}^{*}$ is either an intuitionistic fuzzy conjunction, disjunction, or implication.

Let us consider a pair of intuitionistic fuzzy connectives consisting of an implication $\mathcal{I}^{*}$ on $\mathbb{L}^{*}$ and a conjunction $\mathcal{C}^{*}$ on $\mathbb{L}^{*}$ such that $\mathcal{C}^{*}$ is the dual of $\mathcal{I}^{*}$ with respect to some negation $\mathcal{N}^{*}$ on $\mathbb{L}^{*}$. Consequently, we have $\mathcal{C}^{*}(\mathbf{w}, \mathbf{u})=\mathcal{N}^{*}\left(\mathcal{I}^{*}\left(\mathbf{w}, \mathcal{N}^{*}(\mathbf{u})\right)\right)$ for all $\mathbf{w}, \mathbf{u} \in \mathbb{L}^{*}$ which implies

$$
\begin{aligned}
& \mathcal{C}(\mathbf{x}, \mathbf{y})=\phi^{-1}\left(\mathcal{C}^{*}(\phi(\mathbf{x}), \phi(\mathbf{y}))\right) \\
& =\phi^{-1}\left(\mathcal{N}^{*}\left(\mathcal{I}^{*}\left(\phi(\mathbf{x}), \mathcal{N}^{*}(\phi(\mathbf{y}))\right)\right)\right), \forall \mathbf{x}, \mathbf{y} \in \mathbb{L}^{I}
\end{aligned}
$$

Replacing $\mathcal{I}^{*}$ by $\mathcal{M}^{-1}(\mathcal{I})$ and $\mathcal{N}^{*}$ by $\mathcal{P}^{-1}(\mathcal{N})$ leads to the desired equalities $\mathcal{C}(\mathbf{x}, \mathbf{y})=\mathcal{N}(\mathcal{I}(\mathbf{x}, \mathcal{N}(\mathbf{y})))$ for all $\mathbf{x}, \mathbf{y} \in \mathbb{L}^{I}$. The proof of the converse direction of the theorem concerning the statement "if $\mathcal{C}$ and $\mathcal{I}$ are dual with respect to $\mathcal{N}$ then $\mathcal{C}^{*}$ and $\mathcal{I}^{*}$ are dual with respect to $\mathcal{N}^{*}$ " is similar.

Finally, similar arguments can be applied to pairs of intuitionistic fuzzy connectives consisting of a conjunction and a disjunction or an implication and a disjunction. 


\section{Proof of Theorem 11}

The proof is left as an excercise to the reader.

\section{Proof of Theorem 12}

First, note that Theorem 12 can be applied in particular to the special cases where $C=C^{\prime}$ and $I=I^{\prime}$. In these cases, we simpy write $\mathcal{C}_{C}^{r}$ instead of $\mathcal{C}_{C, C^{\prime}}^{r}$ and $\mathcal{I}_{I}^{r}$ instead of $\mathcal{I}_{I, I^{\prime}}^{r}$.

The sufficiency direction of both statements follows immediately by considering intervals of length 0 , i.e. intervals of the form $[x, x]$, which can be identified with elements of $[0,1]$. The necessity direction follows by applying the commutativity properties of the dilations $C$ and $C^{\prime}$ and the erosions $I$ and $I^{\prime}$ at the respective sides of the intervals.

\section{Proof of Theorem 13}

First note that $\mathbf{x} \neq 0_{\mathbb{I}} I$ if and only if $x_{2} \neq 0$. Consequently, for all $\mathbf{x} \neq 0_{\mathbb{L}^{I}}$ we have that $0<C\left(1, x_{2}\right)$ which implies that $\mathcal{C}_{C}^{p}\left(1_{\mathbb{L}^{I}}, \mathbf{x}\right)>$ $0_{\mathbb{U} I}$ and thus Theorem 1 can be applied to form the $R$-implication of the conjunction $\mathcal{C}_{C}^{p}$. Let $\mathcal{I}$ denote the $R$-implication of the conjunction $\mathcal{C}_{C}^{p}$. In other words, $\mathcal{I}(\mathbf{z}, \mathbf{y})$ is given as follows for all $\mathbf{z}, \mathbf{y} \in \mathbb{L}^{I}$ :

$$
\begin{aligned}
\mathcal{I}(\mathbf{z}, \mathbf{y})=\bigvee\left\{\mathbf{x} \in \mathbb{L}^{I}: \mathcal{C}_{C}^{p}(\mathbf{z}, \mathbf{x}) \leq \mathbf{y}\right\}= \\
\bigvee\left\{\mathbf{x} \in \mathbb{L}^{I}:\left[C\left(z_{1}, x_{1}\right), C\left(z_{1}, x_{2}\right) \vee C\left(z_{2}, x_{1}\right)\right] \leq\left[y_{1}, y_{2}\right]\right\} \\
=\bigvee\left\{\mathbf{x} \in \mathbb{L}^{I}: C\left(z_{1}, x_{1}\right) \leq y_{1} \text { and } C\left(z_{1}, x_{2}\right) \leq y_{2}\right. \\
\left.\left.\quad \text { and } C\left(z_{2}, x_{1}\right)\right] \leq y_{2}\right\} \\
=\left[\bigvee\left\{x_{1} \in[0,1]: C\left(z_{1}, x_{1}\right) \leq y_{1} \text { and } C\left(z_{2}, x_{1}\right) \leq y_{2}\right\},\right. \\
\left.\quad \bigvee\left\{x_{2} \in[0,1]: C\left(z_{1}, x_{2}\right) \leq y_{2}\right\}\right] \\
=\left[I\left(z_{1}, y_{1}\right) \wedge I\left(z_{2}, y_{2}\right), I\left(z_{1}, y_{2}\right)\right]=\mathcal{I}_{I}^{o}(\mathbf{z}, \mathbf{y}) .
\end{aligned}
$$

Thus the first statement of Theorem 13 holds true. Given this fact, an application of Theorem 12 shows that $\mathcal{I}_{I}^{o}$ and $\mathcal{C}_{C}^{p}$ are adjoint if and only if $I$ and $C$ are adjoint. We omit the proof of the second part of the theorem.

\section{Proof of Theorem 14}

As mentioned before, we have $\mathbf{x} \neq 0_{\mathbb{L}^{I}}$ if and only if $x_{2} \neq 0$. Thus, the following statements are equivalent for fuzzy conjunctions such that $C(x, y) \leq C^{\prime}(x, y)$ for all $x, y \in[0,1]$.

$$
\begin{aligned}
& \mathcal{C}_{C, C^{\prime}}^{r}\left(1_{\mathbb{L}^{I}}, \mathbf{x}\right)>0_{\mathbb{L}^{I}} \forall \mathbf{x} \neq 0_{\mathbb{L}^{I}} \\
& \Leftrightarrow\left[C\left(1, x_{1}\right), C^{\prime}\left(1, x_{2}\right)\right]>0_{\mathbb{L}^{I}} \forall \mathbf{x} \neq 0_{\mathbb{L}^{I}} \\
& \Leftrightarrow C^{\prime}\left(1, x_{2}\right)>0 \forall x_{2} \neq 0 .
\end{aligned}
$$

Therefore, the condition $C^{\prime}(1, x)>0$ for all $x \neq 0$ implies that we can construct the $R$-implication of $\mathcal{C}_{C, C^{\prime}}^{r}$ according to Theorem 1. Equation 68 follows directly from Theorem 1 of [49] and thus we obtain the first statement of Theorem 14.

Under the same conditions, an application of Theorem 12 leads to the second statement of Theorem 14, i.e., $\mathcal{I}_{I, I^{\prime}}^{r}$ and its $R$-conjunction are adjoint if and only if the pairs $(I, C)$ and $\left(I^{\prime}, C^{\prime}\right)$ form adjunctions.

The proof of the second part of the theorem is based on a similar line of reasoning. Note that Theorems 13 and 14 generalize Proposition 5 of [25] and Theorem 6.6 of [28], respectively.

\section{Proof of Theorem 15}

On one hand, suppose that $C$ and $I$ as well as $C^{\prime}$ and $I^{\prime}$ are dual with respect to $N$. If $\mathcal{I}$ denotes the dual of $\mathcal{C}_{C, C^{\prime}}^{r}$ with respect to $\mathcal{N}_{N}$ then we have the following equalities for all $\mathbf{z}, \mathbf{x} \in \mathbb{L}^{I}$ :

$$
\begin{aligned}
\mathcal{I}(\mathbf{z}, \mathbf{x}) & =\mathcal{N}_{N}\left(\mathcal{C}_{C, C^{\prime}}^{r}\left(\mathbf{z}, \mathcal{N}_{N}(\mathbf{x})\right)\right) \\
& =\mathcal{N}_{N}\left(\mathcal{C}_{C, C^{\prime}}^{r}\left(\mathbf{z},\left[N\left(x_{2}\right), N\left(x_{1}\right)\right]\right)\right) \\
& =\mathcal{N}_{N}\left(\left[C\left(z_{1}, N\left(x_{2}\right)\right), C^{\prime}\left(z_{2}, N\left(x_{1}\right)\right)\right]\right) \\
& =\left[N\left(C^{\prime}\left(z_{2}, N\left(x_{1}\right)\right), N\left(C\left(z_{1}, N\left(x_{2}\right)\right)\right)\right)\right] \\
& =\left[I^{\prime}\left(z_{2}, x_{1}\right), I\left(z_{1}, x_{2}\right)\right]=\mathcal{I}_{I^{\prime}, I}^{r}(\mathbf{z}, \mathbf{x}) .
\end{aligned}
$$

On the other hand, the assumption that $\mathcal{C}_{C, C^{\prime}}^{r}$ and $\mathcal{I}_{I^{\prime}, I}^{r}$ are dual with respect to $\mathcal{N}_{N}$ leads to the following identities that are valid for all $\mathbf{z}, \mathbf{x} \in \mathbb{L}^{I}:$

$$
\begin{gathered}
\mathcal{N}_{N}\left(\mathcal{C}_{C, C^{\prime}}^{r}\left(\mathbf{z}, \mathcal{N}_{N}(\mathbf{x})\right)\right)=\mathcal{I}_{I^{\prime}, I}^{r}(\mathbf{z}, \mathbf{x}) \\
\Leftrightarrow\left[N\left(C^{\prime}\left(z_{2}, N\left(x_{1}\right)\right), N\left(C\left(z_{1}, N\left(x_{2}\right)\right)\right)\right)\right] \\
=\left[I^{\prime}\left(z_{2}, x_{1}\right), I\left(z_{1}, x_{2}\right)\right] .
\end{gathered}
$$

Therefore, we conclude that $C$ and $I$ well as $C^{\prime}$ and $I^{\prime}$ are dual with respect to $N$.

\section{Proof of Theorem 16}

On one hand, let us assume that $C$ and $I$ are dual with respect to $N$. If $\mathcal{I}$ denotes the $\mathcal{N}_{N}$-dual implication of $\mathcal{C}_{C}^{p}$ then we obtain the following identities for all $\mathbf{x}, \mathbf{y} \in \mathbb{L}^{I}$ :

$$
\begin{aligned}
& \mathcal{I}(\mathbf{x}, \mathbf{y})=\mathcal{N}_{N}\left(\mathcal{C}_{C}^{p}\left(\mathbf{x}, \mathcal{N}_{N}(\mathbf{y})\right)\right) \\
& =\mathcal{N}_{N}\left(\mathcal{C}_{C}^{p}\left(\mathbf{x},\left[N\left(y_{2}\right), N\left(y_{1}\right)\right]\right)\right) \\
& =\mathcal{N}_{N}\left(\left[C\left(x_{1}, N\left(y_{2}\right)\right), C\left(x_{1}, N\left(y_{1}\right)\right) \vee C\left(x_{2}, N\left(y_{2}\right)\right)\right]\right)= \\
& {\left[N\left(C\left(x_{1}, N\left(y_{1}\right)\right) \vee C\left(x_{2}, N\left(y_{2}\right)\right)\right), N\left(C\left(x_{1}, N\left(y_{2}\right)\right)\right)\right]=} \\
& {\left[N\left(C\left(x_{1}, N\left(y_{1}\right)\right)\right) \wedge N\left(C\left(x_{2}, N\left(y_{2}\right)\right)\right), N\left(C\left(x_{1}, N\left(y_{2}\right)\right)\right)\right]} \\
& =\left[I\left(x_{1}, y_{1}\right) \wedge I\left(x_{2}, y_{2}\right), I\left(x_{1}, y_{2}\right)\right]=\mathcal{I}_{I}^{o}(\mathbf{x}, \mathbf{y}) .
\end{aligned}
$$

On the other hand, if $\mathcal{C}_{C}^{p}$ and $\mathcal{I}_{I}^{o}$ are dual with respect to $\mathcal{N}_{N}$ then we have the following equalities for all $\mathbf{x}, \mathbf{y} \in \mathbb{L}^{I}$ :

$$
\begin{aligned}
& {\left[N\left(C\left(x_{1}, N\left(y_{1}\right)\right)\right) \wedge N\left(C\left(x_{2}, N\left(y_{2}\right)\right)\right), N\left(C\left(x_{1}, N\left(y_{2}\right)\right)\right)\right]} \\
& =\left[I\left(x_{1}, y_{1}\right) \wedge I\left(x_{2}, y_{2}\right), I\left(x_{1}, y_{2}\right)\right] .
\end{aligned}
$$

This fact implies that $C$ and $I$ are dual with respect to $N$. The proof of the second part follows a similar line of reasoning.

\section{References}

1. Atanassov, K. Intuitionistic fuzzy sets. Tech. rep., Bulgarian Academy of Science, Central Techn. Library, 1983.

2. Atanassov, K. Intuitionistic fuzzy sets. Fuzzy Sets and Systems 20, 3 (1986), 87-96.

3. Atanassov, K. Intuitionistic Fuzzy Sets. Physica Verlag, Heidelberg, Germany, 1999.

4. Atanassov, K. Remarks on the conjunctions, disjunctions and implications of the intuitionistic fuzzy logic. International Journal of Uncertainty, Fuzziness and Knowledge-Based Systems 8, 1 (2001), 55-65.

5. AtAnASSOV, K., AND GARgOv, G. Interval-valued intuitionistic fuzzy sets. Fuzzy Sets and Systems 31, 3 (1989), 343-349.

6. Atanassov, K., and Gargov, G. Elements of intuitionistic fuzzy logic. Fuzzy Sets and Systems 95, 1 (1998), 39-52. 
7. Bandler, W., And Kohout, L. Fuzzy power sets and fuzzy implication operators. Fuzzy Sets and Systems 4, 1 (July 1980), 13-30.

8. Bargiela, A., And Pedrycz, W. Granular Computing: An Introduction. Kluwer Academic Publishers, Hingham, MA, 2003.

9. BIRKhoff, G. Lattice Theory, 3 ed. American Mathematical Society, Providence, 1993.

10. Bloch, I. Dilation and erosion of spatial bipolar fuzzy sets. In Applications of Fuzzy Sets Theory, vol. 4578 of Lecture Notes in Computer Science. July 2007, pp. 385-393.

11. BLOCH, I. Bipolar fuzzy mathematical morphology for spatial reasoning. In Mathematical Morphology and Its Application to Signal and Image Processing, vol. 5720 of Lecture Notes in Computer Science. 2009, pp. 24-34.

12. BLoch, I. Duality vs. adjunction for fuzzy mathematical morphology and general form of fuzzy erosions and dilations. Fuzzy Sets and Systems 160, 13 (2009), 1858-1867.

13. BLOCH, I. Lattices of fuzzy sets and bipolar fuzzy sets, and mathematical morphology. Information Sciences (2010). in press, available online.

14. Bloch, I., Heijmans, H., And Ronse, C. Mathematical Morphology. In Handbook of Spatial Logics, M. Aiello, I. PrattHartmann, and J. van Benthem, Eds. Springer, 2007, ch. 14, pp. 857-944.

15. Bloch, I., ANd Maitre, H. Fuzzy mathematical morphologies: a comparative study. Pattern Recognition 28, 9 (1995), 13411387.

16. Braga-Neto, U., And Goutsias, J. Supremal multiscale signal analysis. SIAM Journal of Mathematical Analysis 36, 1 (2004), 94-120.

17. Cornelis, C., Descrijver, G., And Kerre, E. E. Classification of intuitionistic fuzzy implicators: an algebraic approach. In Proceedings of the 6th Joint Conference on Information Sciences (2002), pp. $105-108$.

18. Cornelis, C., Descrijver, G., And Kerre, E. E. Implication in intuitionistic fuzzy and interval-valued fuzzy set theory: Construction, classification, application. International Journal of Approximate Reasoning 35 (2004).

19. Cornelis, C., AND Kerre, E. E. Inclusion measures in intuitionistic fuzzy set theory. Lecture Notes in Computer Science 2711 (2003).

20. DE BAETS, B. Fuzzy morphology: A logical approach. In Uncertainty Analysis in Engineering and Science: Fuzzy Logic, Statistics, and Neural Network Approach, B. M. Ayyub and M. M. Gupta, Eds. Kluwer Academic Publishers, Norwell, 1997, pp. 5367.

21. De Baets, B., Kerre, E., And Gupta, M. The fundamentals of fuzzy mathematical morphology, part 1: basic concepts. International Journal of General Systems 23 (1994), 155-171.

22. De Baets, B., Kerre, E., And Gupta, M. The fundamentals of fuzzy mathematical morphology, part 2: idempotence, convexity and decomposition. International Journal of General Systems 23 (1995), 307-322.

23. Deng, T., And Chen, Y. Generalized fuzzy morphological operators. In Fuzzy Systems and Knowledge Discovery, vol. 3614 of Lecture Notes in Computer Science. 2005, pp. 275-284.

24. Deng, T., And Heijmans, H. Grey-scale morphology based on fuzzy logic. Journal of Mathematical Imaging and Vision 16, 2 (Mar. 2002), 155-171.

25. Deschrijver, G., And Cornelis, C. Representability in interval-valued fuzzy set theory. International Journal of Uncertainty, Fuzziness and Knowledge-Based Systems 15, 3 (2007), 345-361.

26. Deschrijver, G., Cornelis, C., And Kerre, E. E. On the representation of intuitionistic fuzzy t-norms and t-conorms. IEEE Transactions on Fuzzy Systems 12, 1 (2004), 45-61.
27. Deschrijver, G., And Kerre, E. E. On the relationship between some extensions of fuzzy set theory. Fuzzy Sets and Systems 133 (2003), 227-235.

28. DeschriJver, G., AND Kerre, E. E. Implicators based on binary aggregation operators in interval-valued fuzzy set theory. Fuzzy Sets and Systems 153 (2005), 229-248.

29. Deschrijver, G., And Kerre, E. E. Triangular norms and related operators in L*-fuzzy set theory. In Logical, Algebraic, Analytic, and Probabilistic Aspects of Triangular Norms, E. P. Klement and R. Mesiar, Eds. Elsevier, Amsterdam, 2005, ch. 8, pp. 231-259.

30. Deschrijver, G., And Kerre, E. E. On the position of intuitionistic fuzzy set theory in the framework of theories modelling imprecision. Information Sciences 177 (2007), 1860-1866.

31. Dubois, D., GotTwald, S., Hajek, P., Kacprzy, J., And PRADE, H. Terminological difficulties in fuzzy set theory - the case of intuitionistic fuzzy sets. Fuzzy Sets and Systems 156 (December 2005), 485-491.

32. Goetcherian, V. From binary to grey tone image processing using fuzzy logic concepts. Pattern Recognition 12, 1 (Jan. 1980), $7-15$.

33. Goguen, J. L-fuzzy sets. Journal of Mathematical Analysis and Applications 18, 1 (Apr. 1967), 145-174.

34. Grattan-Guinness, I. Fuzzy membership mapped onto interval and many-valued quantities. Zentralblatt der Mathematik. Logik. Grundlagen Math. 22 (1975), 149-160.

35. HARAlick, R., AND Shapiro, L. Computer and Robot Vision, vol. I. Addison-Wesley, New York, 1992.

36. Haralick, R., Sternberg, S., And ZhuAng, X. Image analysis using mathematical morphology: Part I. IEEE Transactions on Pattern Analysis and Machine Intelligence 9, 4 (July 1987), 532-550.

37. Heijmans, H. Morphological Image Operators. Academic Press, New York, NY, 1994.

38. Heijmans, H., And KREShet, R. Inf-semilattice approach to self-dual morphology. Journal of Mathematical Imaging and Vision 17 (2002).

39. Heijmans, H., AND Ronse, C. The algebraic basis of mathematical morphology: I. Dilations and erosions. Computer Vision, Graphics, and Image Processing 50 (1990), 245-295.

40. Herman, G. Image Reconstruction from Projections: The fundamentals of Computerized tomography. Academic Press, New York, NY, 1980.

41. Htun, Y. Y., AND AYE, K. K. Fuzzy mathematical morphology approach in image processing. vol. 42 of World Academy of Science, Engineering and Technology, pp. 659-665.

42. JAHN, K. Interval-wertige Mengen. Math. Nach. 68 (1975), 115132.

43. JAIN, A. Fundamentals of Digital Image Processing. Prentice Hall, Englewood Cliffs, NJ, 1989.

44. KIM, C. Segmenting a low-depth-of-field image using morphological filters and region merging. IEEE Transactions on Image Processing 14, 10 (2005), 1503-1511.

45. KitAiniK, L. Fuzzy Decision Procedures with Binary Relations. Kluwer Academic Publishers, 1993.

46. Maragos, P. Lattice image processing: A unification of morphological and fuzzy algebraic systems. Journal of Mathematical Imaging and Vision 22, 2-3 (May 2005), 333-353.

47. Matheron, G. Elements Pour une Theorie des Milieux Poreux. Masson, Paris, 1967.

48. Matheron, G. Random Sets and Integral Geometry. Wiley, New York, 1975.

49. Medina, J. Adjoint pairs on interval-valued fuzzy sets. In Applications of Information Processing and Uncertainty in KnowledgeBased Systems, Proceedings of IPMU 2010 (Dortmund, Germany, June 2010), pp. 430-439. 
50. Mendel, J. M. Computing with words: Zadeh, Turing, Popper and Occam. IEEE Computational Intelligence Magazine 2, 4 (Nov. 2007), 10-17.

51. Mendel, J. M., John, R., And LiU, F. Interval type-2 fuzzy logic systems made simple. IEEE Transactions on Fuzzy Systems 14, 6 (Dec. 2006), 808-821.

52. Mendel, J. M., AND Wu, H. Type-2 fuzzistics for symmetric interval type-2 fuzzy sets: Part 1, forward problems. IEEE Transactions on Fuzzy Systems 14, 6 (Dec. 2007), 781-792.

53. Mendel, J. M., AND Wu, H. Type-2 fuzzistics for symmetric interval type-2 fuzzy sets: Part 2, inverse problems. IEEE Transactions on Fuzzy Systems 15, 2 (Apr. 2007), 301-308.

54. Meyer, F. Topographic distance and watershed lines. Signal Processing 38, 1 (July 1994), 113-125.

55. Meyer, F., AND Beucher, S. Morphological segmentation. Journal of Visual Communication and Image Representation 1, 1 (Oct. 1990), 21-45.

56. Nachtegael, M., And Kerre, E. Connections between binary, gray-scale and fuzzy mathematical morphologies. Fuzzy Sets and Systems 124, 1 (Nov. 2001), 73-85.

57. Nachtegael, M., Sussner, P., Melange, T., And Kerre, E. Modelling numerical and spatial uncertainty in grayscale image capture using fuzzy set theory. In Proceedings of the North-American Simulation Technology Conference 2008 (Montreal, Canada, Aug. 2008), pp. 15-22.

58. Nachtegael, M., Sussner, P., Melange, T., And Kerre, E. Some aspects of interval-valued and intuitionistic fuzzy mathematical morphology. In Proceedings of the 2008 World Congress in Computer Science, Computer Engineering, and Applied Computing (Las Vegas, U.S.A., July 2008), pp. 538-543.

59. Nachtegael, M., Sussner, P., Melange, T., And Kerre, E. On the role of complete lattices in mathematical morphology: From tool to uncertainty model. Information Sciences (2010). in press.

60. NiEWIAdomski, A. Interval-valued and interval type-2 fuzzy sets: A subjective comparison. In Proceedings of the 2007 IEEE International Conference on Fuzzy Systems (July 2007), pp. 11981203.

61. Pedrycz, W., And Gomide, F. An Introduction to Fuzzy Sets: Analysis and Design. MIT Press Complex Adaptive Systems, 1998.

62. Pitas, I., And Venetsanopoulos, A. Morphological shape decomposition. IEEE Transactions on Pattern Analysis and Machine Intelligence 12, 1 (1990), 38-45.

63. Roerdink, J., AND Meijster, A. The watershed transform: definitions, algorithms, and parallelization strategies. Fundamenta Informaticae 41 (2000), 187i£ ; -228.

64. Ronse, C. Why mathematical morphology needs complete lattices. Signal Processing 21, 2 (Oct. 1990), 129-154.

65. Ronse, C., AND SERRA, J. Algebraic foundations of morphology. In Mathematical morphology: from theory to applications, L. Najman and H. Talbot, Eds. ISTE / J. Wiley \& Sons, 2010, ch. 2 , pp. $35-80$.

66. Sambuc, R. Fonctions $\Phi$ - floues. Application l'aide au diagnostic en pathologie tyroidienne. $\mathrm{PhD}$ thesis.

67. SERRA, J. Introduction a la morphologie mathematique. Booklet no. 3, Cahiers du Centre de Morphologie Mathematique, Fontainebleau, France, 1969.

68. Serra, J. Image Analysis and Mathematical Morphology. Academic Press, London, 1982

69. Serra, J. Image Analysis and Mathematical Morphology, Volume 2: Theoretical Advances. Academic Press, New York, 1988.

70. Sinha, D., AND Dougherty, E. R. A general axiomatic theory of intrinsically fuzzy mathematical morphologies. IEEE Transactions on Fuzzy Systems 3, 4 (November 1995), 389-403.

71. Sinha, D., AND Dougherty, R. Fuzzification of set inclusion: theory and applications. Fuzzy Sets and Systems 55, 1 (April 1993), 15-42.
72. Sinha, D., Sinha, P., Dougherty, E., and Batman, S. Design and analysis of fuzzy morphological algorithms for image processing. IEEE Transactions on Fuzzy Systems 5, 4 (Nov. 1997), 570-583.

73. Sobania, A., And Evans, J. P. O. Morphological corner detector using paired triangular structuring elements. Pattern Recognition 38, 7 (2005), 1087-1098.

74. SoILle, P. Morphological Image Analysis. Springer Verlag, Berlin, 1999.

75. Sternberg, S. Grayscale morphology. Computer Vision, Graphics and Image Processing 35 (1986), 333-355.

76. SUSSNER, P., AND ESMI, E. Morphological perceptrons with competitive learning: Lattice-theoretical framework and constructive learning algorithm. Information Sciences (2010). in press.

77. Sussner, P., AND VAlle, M. E. Implicative fuzzy associative memories. IEEE Transactions on Fuzzy Systems 14, 6 (2006), 793-807.

78. Sussner, P., AND VAlle, M. E. Recall of patterns using morphological and certain fuzzy morphological associative memories. In Proceedings of the IEEE World Conference on Computational Intelligence 2006 (Vancouver, Canada, 2006).

79. Sussner, P., AND VAlle, M. E. Morphological and certain fuzzy morphological associative memories for classification and prediction. In Computational Intelligence Based on Lattice Theory, V. Kaburlassos and G. X. Ritter, Eds., vol. 67. Springer Verlag, Heidelberg, Germany, 2007, pp. 149 - 173.

80. Sussner, P., AND VAlle, M. E. Classification of fuzzy mathematical morphologies based on concepts of inclusion measure and duality. Journal of Mathematical Imaging and Vision 32, 2 (2008), 139-159.

81. Sussner, P., And Valle, M. E. Fuzzy associative memories and their relationship to mathematical morphology. In Handbook of Granular Computing, W. Pedrycz, A. Skowron, and V. Kreinovich, Eds. John Wiley and Sons, Inc., New York, 2008, ch. 33 .

82. Tretiak, O., AND MetZ, C. E. The exponencial radon transform. SIAM J. Appl. Math. 39 (1980), 341-354.

83. TÜRKŞEN, I. B. Knowledge representation and approximate reasoning with type 2 fuzzy sets. In Proceedings of the IEEE International Conference on Fuzzy Systems (Mar. 1995), pp. 1911-1917.

84. UNCU, Ö., AND TÜRKȘEN, I. B. Discrete interval type 2 fuzzy system models using uncertainty in learning parameters. IEEE Transactions on Fuzzy Systems 15, 1 (2007), 90-106.

85. VALLE, M. E., AND SUSSNER, P. A general framework for fuzzy morphological associative memories. Fuzzy Sets and Systems 159, 7 (2008), 747-768.

86. Vince, L., AND SoILle, P. Watersheds in digital spaces: an efficient algorithm based on immersion simulations. IEEE Transactions on Pattern Analysis and Machine Intelligence 13, 6 (June 1991), 583-598

87. WANG, G., AND HE, Y. Intuitionistic fuzzy sets and L-fuzzy sets. Fuzzy Sets and Systems 110, 2 (2000), 271-274.

88. ZADEH, L. A. Fuzzy sets. Information and Control 8, 3 (1965), 338-353.

89. ZADEH, L. A. The concept of a linguistic variable and its application to approximate reasoning - i. Information Sciences 8 (1975), 199-249.

90. ZADEH, L. A. Toward a theory of fuzzy information granulation and its centrality in human reasoning and fuzzy logic. Fuzzy Sets and Systems 90 (1997), 111-127.

91. ZHANG, W.-R. Bipolar fuzzy sets and relations: a computational framework for cognitive modeling and multiagent decision analysis. In NAFIPS/IFIS/NASA '94 (Dec. 1994), pp. 305-309.

92. ZHANG, W.-R., AND ZHANG, L. YinYang bipolar logic and bipolar fuzzy logic. Information Sciences 165, 3-4 (October 2004), 265-287. 